\title{
Finite-volume hyperbolic 3-manifolds contain immersed quasi-Fuchsian surfaces
}

\author{
MARK D BAKER \\ DARYL COOPER
}

\begin{abstract}
The paper contains a new proof that a complete, non-compact hyperbolic 3-manifold with finite volume contains an immersed, closed, quasi-Fuchsian surface.
\end{abstract}

57M50, 20F65; 20F67

A complete finite-volume hyperbolic 3-manifold with cusps is a non-compact hyperbolic 3 -manifold with finite volume and universal cover hyperbolic space. We give a new proof of the following result of Masters and Zhang [11; 12].

Theorem 0.1 Suppose $M$ is a complete finite-volume hyperbolic 3-manifold with cusps. Then there is a $\pi_{1}$-injective immersion $f: S \rightarrow M$ of a closed, orientable surface $S$ with genus at least 2 such that $f_{*}\left(\pi_{1} S\right)$ is a quasi-Fuchsian subgroup of $\pi_{1} M$.

Cooper, Long and Reid [6] showed that such manifolds contain geometrically finite closed surface groups but there might be accidental parabolics. Kahn and Markovic [9] have shown that a closed hyperbolic 3-manifold contains an immersed QF (quasiFuchsian) surface.

A prefabricated 3-manifold $Z$ is the union of a finite number of convex pieces, each of which is either a rank- 2 cusp or a $\mathrm{QF}$ manifold $Q_{i}$ with rank-1 cusps. We require simple combinatorics: there are exactly two rank- 1 cusps, with slopes that intersect once, inside each rank- 2 cusp of $Z$. See Definition 1.1 for the precise definition. The convex combination theorem (Baker and Cooper [2]) is used to ensure $Z$ has a convex thickening $\mathrm{CH}(Z)$. In this case $\partial Z$ consists of closed incompressible surfaces without parabolics. The main theorem follows from Theorem 1.2, which says there is a covering space of $M$ that has a convex core that is a prefabricated manifold. This construction of QF surfaces is similar to the method used in Baker and Cooper [2], and Cooper and Long [5].

The crucial step is to control how the QF manifold pieces of $Z$ intersect. In Section 3 we study the intersection $Q_{1} \cap Q_{2}$ of two QF manifolds with cusps. This is governed by a finite collection of convex subsurfaces immersed by local isometries into $\partial Q_{i}$. A 
compact core of $Q_{i}$ is homeomorphic to an interval times a compact surface $F$. A spider is a compact subsurface $X \subset F$ satisfying certain conditions. After taking finite covers, each component of $Q_{1} \cap Q_{2}$ is described by a spider.

The crucial step relies on a result about surfaces: the spider theorem 2.5. Each component of $X \cap \partial F$ is an arc called a foot of the spider $X$. We show that if every component of $\partial F$ contains at least one spider foot then, after replacing $F$ by a suitable finite cover of $F$, and choosing certain lifts of the spiders, every boundary component of $F$ contains exactly one spider foot. This ensures the above mentioned simple combinatorics for $Z$.

In Section 5 we discuss the relation between our proof and that of Masters and Zhang.

Acknowledgements The authors thank IHP and Universite de Rennes 1 for their hospitality during completion of this work. The authors were partially supported by NSF grant number 1207068; Cooper also by NSF grants 0706887, 1065939, 1045292, and DMS grants 1107452, 1107263, 1107367 "RNMS: GEometric structures And Representation varieties" (the GEAR Network) and the CNRS.

\section{Prefabricated 3-manifolds}

In this section we define prefabricated 3-manifolds and use the convex combination theorem to make them convex. We show the boundary consists of incompressible surfaces without parabolics. First we review some material about convex hyperbolic manifolds; see [2, Section 2] for further discussion.

The following definition is not standard. A hyperbolic manifold is a smooth $n$-manifold, possibly with boundary, equipped with a metric so that every point has a neighborhood that is isometric to a subset of the hyperbolic space $\mathbb{H}^{n}$. An example is a compact annulus in $\mathbb{H}^{2}$. A connected hyperbolic $n$-manifold $M$ is convex if every pair of points in the universal cover $\widetilde{M}$ are connected by a geodesic. It is complete if the universal cover is isometric to $\mathbb{H}^{n}$, and metrically complete if every Cauchy sequence converges. If a hyperbolic $n$-manifold $M$ is convex, then the developing map embeds $\widetilde{M}$ isometrically into $\mathbb{H}^{n}$, the covering transformations of $\widetilde{M}$ extend to give a group $\Gamma$ of isometries of $\mathbb{H}^{n}$, and $M$ is isometric to a submanifold of $\mathbb{H}^{n} / \Gamma$. If $M$ is convex and $f: M \rightarrow N$ is a local isometry into a hyperbolic $n$-manifold $N$, then $f$ is $\pi_{1}$-injective.

A hyperbolic $n$-manifold $N$ is a thickening of a connected hyperbolic $n$-manifold $M$ if $M \subset N$ and $\operatorname{incl}_{*}: \pi_{1} M \rightarrow \pi_{1} N$ is an isomorphism. If, in addition, $N$ is convex then $N$ is called a convex thickening of $M$. 
If $M$ is a subset of a metric space $N$, the $\kappa$-neighborhood of $M$ in $N$ is

$$
\mathcal{N}_{K}(M ; N)=\{x \in N: d(x, M) \leq \kappa\} .
$$

If $M$ is a disjoint union of convex hyperbolic manifolds $M_{i}$, and $\kappa \geq 0$, the $\kappa-$ thickening of $M$ is the disjoint union of the convex thickenings of the components:

$$
T h_{\kappa}(M)=\bigsqcup_{i} \mathcal{N}_{\kappa}\left(\tilde{M}_{i} ; \mathbb{H}^{n}\right) / \pi_{1} M_{i}
$$

A horocusp is $C=\mathcal{B} / \Gamma$, where $\mathcal{B} \subset \mathbb{H}^{3}$ is a horoball and $\Gamma$ is a discrete, rank-2 free abelian group of parabolics that preserve $\mathcal{B}$. Thus $\partial C=\partial \mathcal{B} / \Gamma$ is a horotorus.

A finite-area Fuchsian group is a subgroup $\Gamma_{F} \subset \operatorname{Isom}\left(\mathbb{H}^{2}\right)$ such that $F=\mathbb{H}^{2} / \Gamma_{F}$ is an orientable, hyperbolic surface with finite area. This is sometimes called a finitely generated Fuchsian group of the first kind. Throughout this paper Fuchsian groups have finite area. An essential loop in a hyperbolic surface is peripheral if it is freely homotopic into the boundary, or into a cusp. Since $F$ has finite area, every peripheral loop in $F$ has parabolic holonomy.

We fix an embedding $\mathbb{H}^{2} \subset \mathbb{H}^{3}$ and use this to identify $\operatorname{Isom}\left(\mathbb{H}^{2}\right)$ with a subgroup of $\operatorname{Isom}\left(\mathbb{H}^{3}\right)$. Then there is a corresponding Fuchsian 3-manifold $M_{F}=\mathbb{H}^{3} / \Gamma_{F}$ that contains $F$ as a totally geodesic surface.

A $Q F$ (quasi-Fuchsian) group is a subgroup $\Gamma \subset \operatorname{Isom}\left(\mathbb{H}^{3}\right)$ such that $M_{\Gamma}=\mathbb{H}^{3} / \Gamma$ is a hyperbolic 3-manifold that is bilipschitz homeomorphic to a Fuchsian 3-manifold. A 3-manifold is $Q F$ if it is convex and the holonomy is a $\mathrm{QF}$ group.

Definition 1.1 A prefabricated manifold is a connected, metrically complete, finitevolume, hyperbolic 3-manifold

$$
Z=\mathcal{C} \cup \mathcal{Q}_{1} \cup \mathcal{Q}_{2} .
$$

Each component of $\mathcal{Q}_{i}$ and of $\mathcal{C}$ is a convex hyperbolic 3-manifold called a piece. Each component of $\mathcal{Q}_{i}$ is a QF 3-manifold with at least one cusp. Each component of $\mathcal{C}$ is a horocusp. These pieces satisfy the following conditions for $i \in\{1,2\}$ and for each component $C$ of $\mathcal{C}$ :

(P1) $\mathcal{Q}_{i} \cap \mathcal{C}$ is the disjoint union of all the cusps in $\mathcal{Q}_{i}$.

(P2) $\mathcal{Q}_{i} \cap \partial C$ is an annulus with core curve $\alpha_{i}(C)$.

(P3) $\alpha_{1}(C)$ intersects $\alpha_{2}(C)$ once transversally.

(P4) Each component of $\mathcal{Q}_{1} \cap \mathcal{Q}_{2}$ intersects $\mathcal{C}$. 
In general, $Z$ is not isometric to a submanifold of $\mathbb{H}^{3} / \Gamma$ for any Kleinian group $\Gamma$. Under additional hypotheses $Z$ has a convex thickening (Corollary 1.4). A complete prefabricated manifold is a complete hyperbolic 3-manifold that is a convex thickening of a prefabricated manifold. The following is an immediate consequence of Theorem 4.2.

Theorem 1.2 Suppose $M$ is a finite-volume hyperbolic 3-manifold with cusps. Then $M$ has a covering space that is a complete prefabricated manifold.

The main theorem Theorem 0.1 follows from this and the fact that a complete prefabricated manifold contains a surface group without parabolics (Proposition 1.6). This gives a surface subgroup of $\pi_{1} M$ that is not a virtual fiber because $M$ has cusps. Since it has no parabolics it is QF by Theorem 1.7. This theorem can also be used to give another proof of the fact $[2,9.4]$ that for every essential simple closed curve $C \subset T$, where $T \subset M$ is a horotorus, there is an essential immersed surface in $M$ bounded by two copies of a finite cover of $C$.

The geodesic compactification of $\mathbb{H}^{n}$ is the closed ball $\overline{\mathbb{H}}^{n}=\mathbb{H}^{n} \sqcup \partial \mathbb{H}^{n}$, where $\partial \mathbb{H}^{n}=S_{\infty}^{n-1}$. The limit set of a subset $A \subset \mathbb{H}^{n}$ is $\Lambda(A)=\operatorname{cl}(A) \cap \partial \mathbb{H}^{n}$ and the convex core $\operatorname{Core}(A) \subset \mathbb{H}^{n}$ of $A$ is the convex hull of $\Lambda(A)$. Thus $\operatorname{Core}(A)$ is empty if and only if $\Lambda(A)$ contains at most one point. Moreover, if $A$ is convex then $\operatorname{Core}(A) \subset \bar{A}$.

If $M$ has a convex thickening, then the convex core of $M$ is $\operatorname{Core}(M)=\operatorname{Core}(\widetilde{M}) / \pi_{1} M$ and the convex hull $\mathrm{CH}(M)$ of $M$ is the smallest convex manifold containing $M$. A hyperbolic manifold $M$ is geometrically finite (Bowditch [4]) if for all (or some) $\delta>0$ the $\delta$-thickening of $\operatorname{Core}(M)$ has finite volume.

Suppose that $N$ is a hyperbolic manifold and $M \subset N$ is a submanifold. Given $\kappa>0$ we say that $N$ contains a $\kappa$-neighborhood of $M$ if $\exp _{p}(v) \in N$ for every $p \in M$ and tangent vector $v \in T_{p} M$ with $\|v\| \leq \kappa$. The next result gives conditions that ensure that a 3-manifold $M=M_{1} \cup M_{2}$, which is the union of two convex hyperbolic submanifolds $M_{1}$ and $M_{2}$, has a convex thickening:

Theorem 1.3 (Convex combination theorem) Suppose the following:

(C1) $Y=Y_{1} \cup Y_{2}$ is a connected hyperbolic 3-manifold that is the union of two convex 3-submanifolds $Y_{1}$ and $Y_{2}$.

(C2) $\quad M=M_{1} \cup M_{2}$ is a connected hyperbolic 3-manifold that is the union of two convex 3-submanifolds $M_{1}$ and $M_{2}$.

(C3) $Y_{i}$ is a thickening of $M_{i}$. 
(C4) $Y$ contains an 8-neighborhood of $M$.

(C5) $Y_{i}$ contains an 8-neighborhood of $M_{i} \backslash\left(M_{1} \cap M_{2}\right)$.

(C6) No bumping: Every component of $Y_{1} \cap Y_{2}$ contains a point of $M_{1} \cap M_{2}$.

Then $M$ has a convex thickening and $\mathrm{CH}(M) \subset N_{8}(M) \subset Y$.

Proof By [2, Theorem 2.9], $M$ has a convex thickening. Hence there is an isometric embedding of the universal cover $\widetilde{M} \subset \mathbb{H}^{3}$. Claim (2.2) in the proof of that theorem establishes that if a geodesic segment $\gamma$ has endpoints in $\widetilde{M}$, then $\gamma \subset N_{6}(\widetilde{M})$. By Lemma 3.11 below, $\mathrm{CH}(\widetilde{M}) \subset N_{2}\left(N_{6}(\widetilde{M})\right)$. It follows that $\mathrm{CH}(M) \subset N_{8}(M)$.

We use this to show that if a prefabricated manifold $Z$ is contained in a much larger one that is made of thickenings of the pieces in the original, then $Z$ has a convex thickening. The number of connected components of a space $X$ is denoted $|X|$.

Corollary 1.4 Suppose $\kappa \geq 8 k$, where $k=\left(|\mathcal{C}|+\left|\mathcal{Q}_{1}\right|+\left|\mathcal{Q}_{2}\right|-1\right)$, and that:

(Z1) $Z^{\kappa}=\mathcal{C}^{\kappa} \cup \mathcal{Q}_{1}^{\kappa} \cup \mathcal{Q}_{2}^{\kappa}$ is a prefabricated manifold.

(Z2) $Z=\mathcal{C} \cup \mathcal{Q}_{1} \cup \mathcal{Q}_{2}$ is a prefabricated manifold contained in $Z^{\kappa}$.

(Z3) $\mathcal{Q}_{i}^{\kappa}$ is a thickening of $\mathcal{Q}_{i}$.

(Z4) $\mathcal{C}^{\kappa}=\operatorname{Th}_{\kappa}(\mathcal{C})$.

(Z5) $\mathcal{Q}_{i}^{\kappa}$ contains a $\kappa$-neighborhood of $\mathcal{Q}_{i} \backslash \mathcal{C}$.

(Z6) Every component of $\mathcal{Q}_{1}^{\kappa} \cap \mathcal{Q}_{2}^{\kappa}$ contains a point of $\mathcal{Q}_{1} \cap \mathcal{Q}_{2}$.

Then $Z$ has a convex thickening that is a submanifold of $Z^{\kappa}$.

Proof There is a hyperbolic 3 -manifold $\mathcal{P}_{1}$ whose components are convex

$$
\mathcal{Q}_{1} \cup \mathcal{C} \subset \mathcal{P}_{1} \subset N_{8|\mathcal{C}|}\left(\mathcal{Q}_{1} \cup \mathcal{C}\right) \subset Z^{\kappa}
$$

obtained by gluing the components of $\mathcal{C}$ (which are rank- 2 cusps) onto the rank- 1 cusps in $\mathcal{Q}_{1}$ one at a time, and taking the convex hull of the result each time. This involves applying Theorem $1.3|\mathcal{C}|$ times. Each time we attach a cusp requires we thicken by 8 , thus $\mathcal{P}_{1} \subset N_{8|\mathcal{C}|}\left(\mathcal{Q}_{1} \cup \mathcal{C}\right)$. It is routine to check the hypotheses of Theorem 1.3 are satisfied at each step.

(P1) and (P2) imply each cusp of $\mathcal{Q}_{1}$ is contained in a unique component of $\mathcal{C}$, and each component of $\mathcal{C}$ contains a unique cusp of $\mathcal{Q}_{1}$. By (Z3) and (Z5) the components of $\mathcal{Q}_{1} \backslash \mathcal{C}$ are far apart, so each component of $\mathcal{P}_{1}$ is a thickening of a single QF manifold in $\mathcal{Q}_{1}$ with a rank-2 cusp glued onto each rank-1 cusp. 
Next do the same for $\mathcal{Q}_{2}$ with another copy of $\mathcal{C}$ to produce $\mathcal{P}_{2}$ with

$$
\mathcal{Q}_{2} \cup \mathcal{C} \subset \mathcal{P}_{2} \subset N_{8|\mathcal{C}|}\left(\mathcal{Q}_{2} \cup \mathcal{C}\right) \subset Z^{\kappa} .
$$

The final step is to glue the components of $\mathcal{P}_{1}$ and $\mathcal{P}_{2}$ together. Clearly $\left|\mathcal{P}_{i}\right|=\left|\mathcal{Q}_{i}\right|$, so this involves applying Theorem $1.3\left(\left|\mathcal{Q}_{1}\right|+\left|\mathcal{Q}_{2}\right|-1\right)$ times. Since $Z$ is connected we can enumerate the connected components of $\mathcal{P}_{1} \sqcup \mathcal{P}_{2}$ in a sequence so that the union of the components in every initial segment of the enumeration is connected.

Inductively on $m$, we have a connected convex manifold $M_{1} \subset N_{8(|\mathcal{C}|+m-1)}(Z)$ that contains the first $m$ components in the enumeration, and set $M_{2}$ equal to the $(m+1)^{\text {th }}$ component. We apply Theorem 1.3 with $Y_{1}=N_{8}\left(M_{1}\right) \subset Z^{\kappa}$ and $Y_{2}=N_{8}\left(M_{2}\right) \subset Z^{\kappa}$. These are convex thickenings by Lemma 1.5, hence properties (C1)-(C5) hold. The no bumping property (C6) in Theorem 1.3 follows from (Z6) and (P4). Then $M_{1} \cup M_{2}$ has a convex thickening $\mathrm{CH}\left(M_{1} \cup M_{2}\right) \subset N_{8}\left(M_{1} \cup M_{2}\right) \subset N_{8(|\mathcal{C}|+m)}(Z)$.

The proof of the following lemma is routine.

Lemma 1.5 Suppose $M \subset N$ are convex hyperbolic 3-manifolds and $N$ is a thickening of $M$ and $N_{8}(\mathrm{CH}(M)) \subset N$. Then $N_{8}(\mathrm{CH}(M)) \cong N_{8}(\mathrm{CH}(\widetilde{M})) / \pi_{1} M$ is a convex thickening of $M$.

Recall that a group is freely indecomposable, or f.i., if it is not the free product of two non-trivial groups.

Proposition 1.6 If $Z$ is a prefabricated manifold, then $\partial Z$ is non-empty and each component is a closed incompressible surface of genus at least 2. Moreover no essential loop in $\partial Z$ is homotopic into a cusp of $Z$.

Proof The boundary of $Z$ contains a non-empty subset of $\partial \mathcal{Q}_{i}$ so is not empty. If $\partial Z$ is compressible then $\pi_{1} Z$ is the free product of two non-trivial groups. We now show it is not.

By Kurosh's theorem [10], the free product of two f.i. groups, neither of which is cyclic, amalgamated along a non-trivial subgroup is f.i., as is an HNN extension of a non-cyclic f.i. group along a non-trivial subgroup.

A tubed surface [2] is a 2-complex formed by gluing a torus onto each boundary component of a compact surface, with nonempty incompressible boundary, so that each boundary component is glued onto an essential simple closed curve in a distinct torus. The fundamental group of a tubed surface is f.i. (exercise for the reader) and not cyclic. 
The prefabricated manifold $Z$ equals $\bigcup_{i} Y_{i}$, where each $Y_{i}$ is homotopy equivalent to a tubed surface. Each component $X$ of $\mathcal{Q}_{1} \cap \mathcal{Q}_{2}$ is convex, thus $\pi_{1}$-injective. Each component $R \subset Y_{i} \cap Y_{j}$ is formed by adding rank-2 cusps to some such $X$, and is thus $\pi_{1}$-injective. Moreover $\pi_{1}(R)$ contains a $\mathbb{Z}^{2}$ subgroup, and is thus not trivial. The gluings result in HNN extensions and amalgamated free products. Hence $\pi_{1} Z$ is f.i..

Suppose there is an essential annulus $A$ in $Z \backslash \operatorname{int}(\mathcal{C})$ with boundary $\partial A=\alpha \sqcup \beta$, where $\alpha \subset \partial Z$ and $\beta \subset \partial C$ for some horocusp $C \subset \mathcal{C}$. By (P2), $\mathcal{Q}_{i} \cap \partial C$ is an annulus and by (P3) the core curves $\alpha_{1}(C)$ and $\alpha_{2}(C)$ of these annuli have intersection number one. It follows that $\beta$ has intersection number $n \neq 0$ with at least one of these core curves. However $[\alpha]=[\beta] \in H_{1}(Z)$ and $n$ depends only on the homology class. Since $\alpha$ is disjoint from these surfaces, $n=0$, which contradicts the existence of $A$.

The next result follows from work of Bonahon and Thurston.

Theorem 1.7 Suppose $M$ is a complete hyperbolic 3-manifold with finite volume, and $S$ is a closed, orientable surface with $\chi(S)<0$ that is $\pi_{1}$-injectively immersed in $M$. Then either $S$ is a virtual fiber, or else $S$ is geometrically finite, in which case either it is QF or some element of $\pi_{1} S$ is (an accidental) parabolic.

\section{Coverings of surfaces containing immersed subsurfaces}

A spider pattern Definition 2.6 consists of a pair of surfaces (possibly not connected) each equipped with various immersed surfaces that are identified in pairs, and is used later to model how QF 3-manifolds intersect. The main result of this section, Theorem 2.8, asserts the existence of a finite cover of a spider pattern with certain properties. This follows easily from Theorem 2.5 whose proof occupies the bulk of this section.

A path in a surface $F$ with endpoints in $\partial F$ is essential if it is not homotopic rel endpoints into the boundary of $F$. A loop in $F$ is peripheral if it is freely homotopic into $\partial F$. A function $f: X \rightarrow Y$ between metric spaces is a local isometry if $X$ has an open cover such that the restriction of $f$ to each set in the open cover is an isometry onto its image.

Definition 2.1 An immersed spider is a triple $(F, X, f)$, where $F$ and $X$ are compact, convex, hyperbolic surfaces, $f: X \rightarrow F$ is a local isometry and:

(I1) Each component of $f^{-1}(\partial F)$ is an arc (called a foot of the spider).

(I2) $X$ has at least 2 feet. 
(I3) If $\gamma$ is an essential loop in $X$ then $f \circ \gamma$ is not peripheral in $F$.

(I4) If $\gamma$ is an arc in $X$ with endpoints on distinct feet then $f \circ \gamma$ is essential in $F$.

Clearly $f^{-1}(\partial F) \subset \partial X$. A spider is called degenerate if $X$ is a disc with exactly two feet. If $f$ is injective we identify $X$ with $f(X)$ and regard the spider as the subsurface $X \subset F$ and refer to $(F, X)$, or sometimes $X$, as an (embedded) spider.

A spider $X$ can be decomposed as $X=B \cup \mathcal{L}$, where $\mathcal{L}$ is a regular neighborhood of the feet of $X$ and $B$ is the closure of $X \backslash \mathcal{L}$ and is called the spider body. Each component $L$ of $\mathcal{L}$ is a rectangle called a leg of the spider and contains a spider foot in the boundary.

Definition 2.2 An immersed spider surface is $\mathcal{S}=(\mathcal{F}, \mathcal{X}, f: \mathcal{X} \rightarrow \mathcal{F})$ such that:

(S1) Each component of $\mathcal{F}$ and $\mathcal{X}$ is a compact, convex, hyperbolic surface.

(S2) If $X \subset \mathcal{X}$ and $F \subset \mathcal{F}$ are components with $f(X) \subset F$ then $(F, X, f \mid X)$ is an immersed spider.

(S3) (Ample spiders) $f^{-1}(C) \neq \phi$ for each component $C \subset \partial \mathcal{F}$.

If $f$ is injective, we regard $\mathcal{X}$ as a subset of $\mathcal{F}$, and then $(\mathcal{F}, \mathcal{X})$ is called an (embedded) spider surface. We say $\mathcal{S}$ is connected if $\mathcal{F}$ is connected, and in this case $\mathcal{F}$ will often be denoted by $F$. The condition (S3) says each boundary component of $\mathcal{F}$ contains the foot of some spider.

Definition 2.3 An embedded spider surface $(\mathcal{F}, \mathcal{X})$ is called simple if $C \cap \mathcal{X}$ is connected for each boundary component $C \subset \partial \mathcal{F}$.

This means each boundary component of $\mathcal{F}$ contains exactly one spider foot.

Definition 2.4 Suppose $\mathcal{S}=(\mathcal{F}, \mathcal{X}, f)$ and $\widetilde{\mathcal{S}}=(\tilde{\mathcal{F}}, \tilde{\mathcal{X}}, \tilde{f})$ are immersed spider surfaces. A spider cover $(p, q): \widetilde{\mathcal{S}} \rightarrow \mathcal{S}$ of spider degree $d$ consists of covering space maps $p: \widetilde{\mathcal{F}} \rightarrow \mathcal{F}$ and $q: \widetilde{\mathcal{X}} \rightarrow \mathcal{X}$ such that $\widetilde{\mathcal{X}}$ is the disjoint union of $d$ copies of $\mathcal{X}$, and $q$ is the natural projection and the following diagram commutes:




The pair $(p, q)$ is called a spider covering map. For each component $X \subset \mathcal{X}$ the components of $q^{-1}(X)$ are called the lifts of $X$. Observe that if $f$ and $\tilde{f}$ are both injective then, after identifying the spiders with subsurfaces of $\tilde{\mathcal{F}}$ and $\mathcal{F}$, we have $q=p \mid \tilde{\mathcal{X}}$. Since $\mathcal{F}$ need not be connected, $p$ might not have a well-defined degree. It is important to check the condition (S3) ample spiders is satisfied when constructing spider covers.

Theorem 2.5 (Spider theorem) Suppose $\mathcal{S}=(F, \mathcal{X}, f)$ is a connected, immersed, spider surface. Then there is a connected, simple, embedded spider surface $\widetilde{\mathcal{S}}=(\widetilde{F}, \widetilde{\mathcal{X}})$ that spider-covers $\mathcal{S}$ and $\tilde{F} \backslash \tilde{\mathcal{X}}$ is connected and $|\partial \widetilde{F}|$ is even.

Proof By Theorem 2.12 there is a spider cover that is an embedded spider surface. By Theorem 2.16 there is a further cover by a simple spider surface with the required properties.

Definition 2.6 An immersed spider pattern is $P=\left(\mathcal{S}_{1}, \mathcal{S}_{2}, \tau\right)$, where $\mathcal{S}_{i}=\left(\mathcal{F}_{i}, \mathcal{X}_{i}, f_{i}\right)$ is an immersed spider surface and $\tau: \mathcal{X}_{1} \rightarrow \mathcal{X}_{2}$ is a map called the pairing that induces a bijection between components.

In later sections the pairing models how QF 3-manifolds are glued along submanifolds. If $f_{1}$ and $f_{2}$ are both injective we omit them from the notation and refer to an embedded spider pattern or just a spider pattern.

Definition 2.7 A spider pattern $\widetilde{P}=\left(\widetilde{\mathcal{S}}_{1}, \widetilde{\mathcal{S}}_{2}, \tilde{\tau}\right)$ covers an immersed spider pattern $P=\left(\mathcal{S}_{1}, \mathcal{S}_{1}, \tau\right)$ if there are spider covers $\left(p_{i}, q_{i}\right): \widetilde{\mathcal{S}}_{i} \rightarrow \mathcal{S}_{i}$ that are compatible with the pairings in the sense that $q_{2} \circ \tilde{\tau}=\tau \circ q_{1}$.

Given an immersed spider surface $(\mathcal{F}, \mathcal{X}, f)$, each connected component $F \subset \mathcal{F}$ determines an immersed spider surface called a component spider surface $\mathcal{S}_{F}=$ $\left(F, \mathcal{X}_{F}, f \mid \mathcal{X}_{F}\right)$ where $\mathcal{X}_{F}=f^{-1}(F)$. A spider pattern is simple if every component spider surface is simple.

Given $\delta>0$, an immersed spider $\left(F, X^{\delta}, f^{\delta}\right)$ is a $\delta$-thickening of another immersed spider $(F, X, f)$ if $X \subset X^{\delta}$, and $f^{\delta}$ is an extension of $f$ and taking appropriate lifts to universal covers $\tilde{f}^{\delta}\left(\tilde{X}^{\delta}\right)$ contains a $\delta$-neighborhood of $\tilde{f}(\tilde{X})$ in $\tilde{F}$. An immersed spider pattern $P^{\delta}$ is a $\delta$-thickening of another immersed spider pattern $P$ if all the component spider surfaces of $P^{\delta}$ are $\delta$-thickenings of those of $P$. It is routine to check that $\delta$-thickenings always exist. The main result of this section is:

Theorem 2.8 (Spider pattern theorem) Given an immersed spider pattern $P$ there is $d>0$ such that for all $\delta>0$ there is a simple embedded spider pattern $\widetilde{P}^{\delta}$ that spider-covers $P^{\delta}$ with spider degree $d$. 
Proof By Theorem 2.5, for each component $F$ of $\mathcal{F}_{i}$ there is a simple spider surface $\widetilde{\mathcal{S}}(F)=(\widetilde{F}, \tilde{\mathcal{X}})$ that spider-covers the component immersed spider surface $\mathcal{S}_{F}$ given by $F$ with some spider degree $d(F)>0$. Moreover $\widetilde{F} \backslash \tilde{\mathcal{X}}$ is connected and $|\partial \widetilde{F}|$ is even. Let $d$ be the lowest common multiple of all the $d(F)$ for $F$ a component of $\mathcal{F}_{1} \sqcup \mathcal{F}_{2}$. Define $\left(\tilde{\mathcal{F}}_{i}, \tilde{\mathcal{X}}_{i}\right)$ to be the disjoint union of $d / d(F)$ copies of $\mathcal{S}(F)$ as $F$ ranges over components of $\mathcal{F}_{i}$. This determines a spider pattern $\widetilde{P}$ except for the pairing $\tilde{\tau}$. There are obvious covering space projections to $P$. Since every spider in $\mathcal{X}_{i}$ has the same number $d$ of lifts to $\tilde{\mathcal{X}}_{i}$ there is a pairing $\tilde{\tau}$ of the spiders in $\widetilde{P}$ that covers the pairing $\tau$. It only remains to arrange the condition on $\delta$. After replacing $P$ by $\widetilde{P}$ it suffices to prove the theorem in the case $P$ is a simple embedded spider pattern.

Given a simple embedded spider pattern $P$ there is $\delta$-thickening $P^{\delta}$ consisting of immersed spiders. We show there is a simple embedded spider cover $\widetilde{P}^{\delta}$ of $P^{\delta}$ with spider degree 1 that is a conservative cover of each component surface. The spiders in $P^{\delta}$ are immersed, and might intersect. The argument in the first paragraph of the proof of Theorem 2.12 shows there is a conservative cover of each component surface $F$ of $P^{\delta}$ and pairwise disjoint embeddings of these thickened spiders. Doing this for each $F$ gives a spider cover $\widetilde{P}^{\delta}$ of spider degree 1 .

We turn now to the proof of Theorem 2.12. A finite sheeted covering space $\widetilde{F}$ of a compact surface $F$ is conservative if $|\partial \widetilde{F}|=|\partial F|$. A map $f: S \rightarrow F$ is a virtual embedding if there is a finite cover $p: \widetilde{F} \rightarrow F$ and a lift $\widetilde{f}: S \rightarrow \widetilde{F}$ that is an embedding. At various times we wish to lift an immersed surface to a finite cover so it is embedded and does not separate.

Proposition 2.9 Suppose $F$ and $Y$ are two compact, convex hyperbolic surfaces. Suppose $f: Y \rightarrow \operatorname{int}(F)$ is a local isometry and $f_{*}\left(\pi_{1} Y\right)$ contains no peripheral element of $\pi_{1} F$. Then there is a conservative cover $\widetilde{F}$ of $F$ such that $f$ lifts to an embedding $\tilde{f}: Y \rightarrow \widetilde{F}$ and $\widetilde{F} \backslash \tilde{f}(Y)$ is connected.

Proof Choose a basepoint $y \in Y$ and use $x=f(y)$ as the base point for $F$. Define $H=f_{*}\left(\pi_{1}(Y, y)\right) \subset \pi_{1}(F, x)$ and let $p_{Y}: \widetilde{F}_{Y} \rightarrow F$ be the cover corresponding to $H$. The map $f$ is $\pi_{1}$-injective so it lifts to a homotopy equivalence $\tilde{f}_{Y}: Y \rightarrow \widetilde{F}_{Y}$. Since $Y$ and $F$ are convex the developing map embeds the universal covers $\tilde{Y} \subset \widetilde{F} \subset \mathbb{H}^{2}$. But $Y$ and $\widetilde{F}_{Y}$ are the quotient of their universal covers by $H$ and it follows that $\widetilde{f}_{Y}$ is injective.

Let $B \subset \pi_{1}(F, x) \backslash H$ be the set represented by loops based at $x$ of length at most $2 \operatorname{diam}(Y)$. Then $B$ is finite. By the conservative separability theorem [3], there is a conservative cover $p: \widetilde{F} \rightarrow F$ and basepoint $\tilde{x} \in \widetilde{F}$ covering $x$ with the following properties: 
(i) There a compact connected $\pi_{1}$-injective subsurface $S \subset \tilde{F}$ with

$$
p_{*}\left(\pi_{1}(S, \tilde{x})\right)=H .
$$

(ii) $p_{*}\left(\pi_{1}(\tilde{F}, \tilde{x})\right)$ contains no element of $B$.

(iii) $\tilde{F} \backslash S$ is connected

(iv) The covering is conservative.

The existence of $S$ implies $f$ lifts to $\tilde{f}: Y \rightarrow \tilde{F}$ with $\tilde{f}(y)=\tilde{x}$ and we claim $\tilde{f}$ is injective.

Suppose $\tilde{f}(a)=\tilde{f}(b)$. In $Y$ there are paths $\alpha$ starting at $y$ and ending at $a$, and $\beta$ starting at $b$ and ending at $y$ both of length at most $\operatorname{diam}(Y)$. This gives two paths $\tilde{\alpha}=\widetilde{f} \circ \alpha$ and $\widetilde{\beta}=\widetilde{f} \circ \beta$ in $\widetilde{F}$. Then $\widetilde{\alpha} \cdot \widetilde{\beta}$ is a loop in $\widetilde{F}$ based at $\tilde{x}$ and going through $\tilde{f}(a)$. It projects to a loop $\gamma$ in $F$ based at $x$ of length at most $2 \operatorname{diam}(Y)$, so $[\gamma] \in H$. Hence $\gamma$ lifts to a loop $\tilde{\gamma}_{Y}$ in $\tilde{F}_{Y}$ based at $\tilde{f}_{Y}(y)$. Since $\tilde{f}_{Y}$ is injective and covers $\tilde{f}$ this implies $a=b$ so $\widetilde{f}$ is injective as asserted.

It follows that $\tilde{f}(Y)$ is a regular neighborhood of convex core of $S$, and the remaining claims follow from (iii) and (iv).

Lemma 2.10 Suppose $\mathcal{S}_{1}=\left(F, X_{1}, f_{1}\right)$ and $\mathcal{S}_{2}=\left(F, X_{2}, f_{2}\right)$ are immersed spiders. Then there is an immersed spider $(F, X, f)$ called a band sum of $\mathcal{S}_{1}$ and $\mathcal{S}_{2}$ such that $X$ is the union of regular neighborhoods of $X_{1}$ and $X_{2}$ that intersect along an arc. Moreover $f \mid X_{i}=f_{i}$ and each foot of $X$ contains exactly one foot of $X_{1} \sqcup X_{2}$.

Proof There is a rectangle $D$ that maps to a convex neighborhood of a long immersed geodesic arc $\lambda$ connecting $X_{1}$ and $X_{2}$. For a suitable choice of $\lambda$ there is a convex thickening, $X$, of $X_{1} \cup D \cup X_{2}$. Details are left to the reader.

Lemma 2.11 Suppose $\left(F, X^{\prime}, f^{\prime}\right)$ is an immersed spider. Then there is an immersed spider $(F, X, f)$ such that $X^{\prime} \subset X$ and $f \mid X=f^{\prime}$ and:

(E1) Every component of $\partial X$ contains at most one foot of $X^{\prime}$.

(E2) Every component $D$ of $c l\left(X \backslash X^{\prime}\right)$ is a disc and $\partial D \cap \partial X \neq \phi$.

Proof Glue an annulus $A(L)$ onto each leg $L$ of $X^{\prime}$, such that $R=X^{\prime} \cap A(L)$ is a rectangle in $L$ that separates the foot of $L$ from the body of $X^{\prime}$ and the closure of $A(L) \backslash R$ is a disc $D(L)$. The resulting surface satisfies (E1) and (E2). This can be done so that the result has a convex thickening for which there is an isometric immersion of $X$ into $F$ extending $f^{\prime}$. The core curve of $A(L)$ maps to a long immersed geodesic loop in $F$, which is not peripheral, but wraps many times around the boundary component containing the foot of $L$. Details are left to the reader. 
The following implies there is a single conservative cover of a compact hyperbolic surface $F$ such that finitely many immersed spiders in $F$ simultaneously lift to embeddings that are non-separating.

Theorem 2.12 (Embedded spiders) Suppose $\mathcal{S}=\left(F, \mathcal{X}, f_{\mathcal{X}}\right)$ is a connected, immersed, spider surface. There is a connected, embedded spider surface $\widetilde{\mathcal{S}}=(\widetilde{F}, \widetilde{\mathcal{X}})$ that is a spider cover of spider degree 1 of $\mathcal{S}$. Furthermore $|\partial \widetilde{F}|=|\partial F|$ and $\widetilde{F} \backslash \widetilde{\mathcal{X}}$ is connected and $\beta_{1}(\tilde{F})>\beta_{1}(\tilde{\mathcal{X}} \cup \partial \tilde{F})$.

Proof By banding the spiders of $\mathcal{X}$ together using Lemma 2.10 we obtain an immersed spider $\left(F, X^{\prime}, f^{\prime}\right)$ containing $\mathcal{X}$. Let $(F, X, f)$ be the immersed spider surface with $X^{\prime} \subset X$ given by Lemma 2.11. Let $F^{+}$be $F$ union a compact convex collar on each component of $\partial F$. Then $X$ is immersed in the interior of $F^{+}$so by Proposition 2.9 there is a conservative cover $\widetilde{F}^{+}$of $F^{+}$and an embedded lift of $X$ to $\tilde{X} \subset \widetilde{F}^{+}$with $\tilde{F}^{+} \backslash \tilde{X}$ connected. Thus $\tilde{X}$ is an embedded spider in the subsurface $\tilde{F} \subset \tilde{F}^{+}$.

For each foot $A \subset \partial \tilde{X} \cap \partial \tilde{F}$ there is a rectangle $L=L(A) \subset \widetilde{F}^{+}$with one side $A$, and the opposite side of $L$ is an $\operatorname{arc}$ in $\partial \tilde{F}^{+}$. Gluing these onto $X$ gives an embedded spider $\tilde{X}^{+} \supset \widetilde{X}$ in $\tilde{F}^{+}$. These rectangles are the legs of $\tilde{X}^{+}$and $\tilde{X}$ is the body of $\tilde{X}^{+}$. There is a bit of fussing to arrange that $\tilde{X}^{+}$is convex; however, the argument below does not require this.

We claim $\tilde{F} \backslash \tilde{X}$ is connected. There is a homeomorphism of pairs $(\tilde{F}, \tilde{X}) \cong\left(\widetilde{F}^{+}, \tilde{X}^{+}\right)$, so it suffices to show $\widetilde{F}^{+} \backslash \tilde{X}^{+}$is connected. Let $L$ be a leg of $\tilde{X}^{+}$and $B \subset \partial \tilde{X}$ the component that intersects $L$. By Lemma 2.11(E1) $B$ is disjoint from all the other legs of $\tilde{X}^{+}$. The arc $B \backslash L$ connects the two sides of $L$ thus adding $L$ onto $\tilde{X}$ does not disconnect the complement. This proves $\widetilde{F} \backslash \tilde{X}$ is connected.

There is a lift of $X^{\prime} \subset X$ to $\tilde{X}^{\prime} \subset \tilde{X}$ and $\tilde{F} \backslash \tilde{X}^{\prime}$ is connected because, by Lemma 2.11(E2), there is a path connecting every point in $\tilde{X} \backslash \tilde{X}^{\prime}$ to a point $p \in \partial \tilde{X}$. Since $\tilde{X}$ is a spider we may choose $p$ in the interior of $\tilde{F}$. Thus $p$ is connected by an arc in $\widetilde{F} \backslash \tilde{X}^{\prime}$ to a point in the connected set $\widetilde{F} \backslash \tilde{X}$.

There is a lift of the subsurface $\mathcal{X} \subset X^{\prime}$ to $\tilde{\mathcal{X}} \subset \tilde{X}^{\prime} \subset \tilde{F}$ and $\tilde{F} \backslash \tilde{\mathcal{X}}$ is connected because $\tilde{X}^{\prime}$ is obtained by band-summing the components of $\tilde{\mathcal{X}}$ and then taking the convex hull. Shrinking the convex hull and then deleting these bands clearly leaves the complement, $\tilde{F} \backslash \tilde{\mathcal{X}}$, connected.

The condition on $\beta_{1}(\tilde{F})$ can be ensured by using a conservative cover of very large degree $d$. The relation between Euler characteristic and degree of a cover implies we may make $\beta_{1}(\tilde{F})$ as large as we wish. However since the cover is conservative and spider degree 1 it follows that $\beta_{1}(\tilde{\mathcal{X}} \cup \partial \tilde{F})$ is independent of the cover. 
It remains to prove Theorem 2.16. If $F$ is a compact surface with boundary, the capped surface $C(F)=F \cup \mathcal{D}$ is the closed surface obtained by gluing a disc onto each circle component of $\partial F$, and $\mathcal{D}$ is the union of the closed discs. If $\mathcal{X}$ is a disjoint union of spiders embedded in $F$ then each component of $\mathcal{X} \cap \partial F$ is an arc and the capped spiders $C(\mathcal{X})=\mathcal{X} \cup \mathcal{D}$ is a compact subsurface of $C(F)$.

Definition 2.13 The spider graph of a spider surface $\mathcal{S}=(\mathcal{F}, \mathcal{X})$ is a bipartite graph $G=G(\mathcal{S})$ with a spider vertex $v(X)$ for each component $X \subset \mathcal{X}$ and a boundary vertex $v(C)$ for each component $C \subset \partial \mathcal{F}$. There is an edge $e(A)$ for each foot $A \subset \mathcal{X}$. The edge $e(A)$ connects $v(X)$ to $v(C)$ where $X \subset \mathcal{X}$ and $C \subset \partial F$ are the components containing $A$.

Embed $G(\mathcal{S})$ in $C(\mathcal{X})=\mathcal{X} \cup \mathcal{D}$ as follows. If $D$ is a disc component of $\mathcal{D}$ with $\partial D=C$ then the vertex $v(C)$ of $G(\mathcal{S})$ is mapped to a point in $D$. If $X$ is a spider then $v(X)$ is mapped to a point in the spider body $B=B(X)$ of $X$. The edge $e(A)$ in $G(\mathcal{S})$ with endpoints $v(X)$ and $v(C)$ corresponds to the leg $L$ of $X$ with $L \cap C=A$. This edge is mapped to an $\operatorname{arc} \gamma=\beta \cdot \lambda \cdot \delta$ in $C(\mathcal{X})$ that is the union of an $\operatorname{arc} \beta \subset B(X)$ starting at $v(X)$ and ending on $L \cap B(X)$, an arc $\lambda \subset L$ connecting $B(X) \cap L$ and $L \cap C$, and an $\operatorname{arc} \delta \subset D$ connecting $L \cap C$ to $v(C)$. It follows that $\mathcal{S}$ is simple if and only if each component of $G(\mathcal{S})$ contains a single spider vertex.

Lemma 2.14 Suppose $(F, \mathcal{X})$ is an embedded spider surface and $F \backslash \mathcal{X}$ is connected. Then the natural map below is injective:

$$
\sigma: H_{1}(G(F, \mathcal{X}) ; \mathbb{Z} / 2) \rightarrow H_{1}(C(F) ; \mathbb{Z} / 2) \rightarrow H_{1}(C(F) ; \mathbb{Z} / 2) / \operatorname{incl}_{*}\left(H_{1}(\mathcal{X} ; \mathbb{Z} / 2)\right) .
$$

Moreover, if $\beta_{1}(F)>\beta_{1}(\mathcal{X} \cup \partial F)$ then $\sigma$ is not surjective.

Proof Suppose $\beta \in Z_{1}(G(F, \mathcal{X}) ; \mathbb{Z} / 2)$ with $0 \neq[\beta] \in H_{1}(G(F, \mathcal{X}) ; \mathbb{Z} / 2)$. There is an edge $e$ of $G(F, \mathcal{X})$ with coefficient 1 in $\beta$. Let $A \subset \partial \mathcal{X}$ be the foot corresponding to $e$. Since $F \backslash \mathcal{X}$ is connected, there is an embedded loop $\alpha \subset F$ such that $\alpha \cap \mathcal{X}=A$. The algebraic intersection of $[\alpha]$ and $[\beta]$ is 1 thus $0 \neq[\beta] \in H_{1}(C(F) ; \mathbb{Z} / 2)$. Every element of $H_{1}(\mathcal{X} ; \mathbb{Z} / 2)$ has intersection number 0 with $[\alpha]$. This is because $\mathcal{X}$ can be isotoped into its interior and is then disjoint from $\alpha$. Thus $\sigma([\beta]) \notin H_{1}(\mathcal{X} ; \mathbb{Z} / 2)$, so $\sigma$ is injective. We also have

$$
\begin{aligned}
\operatorname{dim}[\operatorname{coker} \sigma] & \geq \beta_{1}(C(F))-\left[\beta_{1}(\mathcal{X})+\beta_{1}(G(F, \mathcal{X}))\right] \\
& \geq \beta_{1}(F)-\left[\beta_{1}(\partial F)+\beta_{1}(\mathcal{X})+\beta_{1}(G(F, \mathcal{X}))\right] \\
& =\beta_{1}(F)-\beta_{1}(\mathcal{X} \cup \partial F) .
\end{aligned}
$$

The additional hypothesis ensures this is positive. 
A morphism between spider graphs is a simplicial map that preserves the type of each vertex, and is an isomorphism if it is also bijective. Let $\ell(G)$ be the number of edges in a shortest circuit in $G$. Since $G$ is bipartite, $\ell(G)$ is even. If there are no circuits, $\ell(G)=\infty$. The boundary of a spider surface $\mathcal{S}=(\mathcal{F}, \mathcal{X})$ is the boundary of the underlying surface: $\partial \mathcal{S}=\partial \mathcal{F}$.

Lemma 2.15 Suppose $\mathcal{S}=(F, \mathcal{X})$ and $\widetilde{\mathcal{S}}=(\widetilde{F}, \tilde{\mathcal{X}})$ are connected, embedded, spider surfaces. Then a spider cover $(p, q): \widetilde{\mathcal{S}} \rightarrow \mathcal{S}$ with spider degree $d$ induces a morphism $p_{G}: G(\widetilde{\mathcal{S}}) \rightarrow G(\mathcal{S})$ and:

(G1) If $d=1$ and $p$ is a conservative cover then $p_{G}$ is an isomorphism.

(G2) If $d=1$ then $\ell(G(\widetilde{\mathcal{S}})) \geq \ell(G(\mathcal{S}))$.

(G3) If $p \mid \partial C$ is injective for each component $C \subset \partial \tilde{F}$ and $\tilde{\mathcal{X}}=p^{-1} \mathcal{X}$, then $p_{G}$ is a covering space projection.

Proof (G1) is obvious. (G3) follows from the fact the spider graph $G(\mathcal{S})$ embeds in $F$ and since both spiders and components of $\partial F$ lift, it follows that we can choose embeddings with $G(\widetilde{\mathcal{S}})=p^{-1}(G(\mathcal{S}))$.

For (G2): if the cover is conservative the result follows from (G1). Otherwise if the cover is not conservative, then $|\partial \widetilde{F}|>|\partial F|$ and $G(\widetilde{\mathcal{S}})$ has more vertices corresponding to components of the boundary than $G(\mathcal{S})$. Clearly $p_{G}$ is a bijection on the interiors of edges and on vertices corresponding to spiders. However if $C$ is a component of $\partial F$ then the pre-image of $v(C)$ has one vertex for each component of $p^{-1}(C)$. One may regard $G(\widetilde{\mathcal{S}})$ as obtained from $G(\mathcal{S})$ by cutting into several pieces some of the vertices of $G(\mathcal{S})$ and attaching the edges to the resulting subdivided vertices in some way.

Theorem 2.16 (Simple spiders) Every connected embedded spider surface $\mathcal{S}=$ $(F, \mathcal{X})$ is spider-covered by a simple spider surface $\widetilde{\mathcal{S}}=(\widetilde{F}, \widetilde{\mathcal{X}})$ such that $\widetilde{F} \backslash \widetilde{\mathcal{X}}$ is connected and $\widetilde{F}$ has an even number of boundary components.

Proof For each component $C$ of $\partial F$ the excess number of spider feet on $C$ is

$$
e(C)=|\mathcal{X} \cap C|-1 \text {. }
$$

Condition (S3), ample spiders, implies $e(C) \geq 0$ for all components $C \subset \partial F$. The excess number of spider feet on $F$ is

$$
e=e(F)=|X \cap \partial F|-|\partial F|=\sum_{C} e(C) .
$$

Observe $e(C)+1$ is degree of the vertex $v(C)$ and is therefore determined by the spider graph $G(\mathcal{S})$. The spider surface is simple if and only if $e=0$. 
Claim Every connected spider surface is spider-covered by a spider surface $\mathcal{S}=$ $(F, \mathcal{X})$ with the following properties:

(F1) $F \backslash \mathcal{X}$ is connected.

(F2) $|\partial F| \geq 4$ and is even.

(F3) $e=e(F)$ is even.

(F4) $\ell(G(F, \mathcal{X}))>4$.

Properties (F2)-(F4) are determined by the isomorphism type of the spider graph $G(F, \mathcal{X})$. Property (F4) says no spider has two feet on the same boundary component (no circuit of length 2) and two spiders have feet on at most one common boundary component (no circuit of length 4).

Proof of claim The cover consists of a sequence of 5 spider covers (A), (B), (A), (B), (A) of two types called (A) and (B). A spider cover preserves property (Fn) if whenever the original spider surface has this property, so does the spider cover.

The type (A) cover is a conservative cover of $F$ given by Theorem 2.12 and thus has property (F1). It is conservative and has spider-degree 1, so by Lemma 2.15(G1) it preserves the isomorphism type of the spider graph, and therefore it preserves the remaining properties.

The type (B) cover is the regular cover $p: \widetilde{F} \rightarrow F$ corresponding to the kernel of the natural surjection

$$
\pi_{1} F \rightarrow H_{1}(C(F) ; \mathbb{Z} / 2) / \operatorname{incl}_{*}\left(H_{1}(\mathcal{X} ; \mathbb{Z} / 2)\right) .
$$

Every spider in $\mathcal{X}$, and each boundary component of $F$, lifts for this cover, and $\tilde{\mathcal{X}}=p^{-1}(\mathcal{X})$ is an ample collection of disjoint spiders in $\tilde{F}$. By Lemma 2.15(G3), the induced morphism

$$
p_{G}: \widetilde{G}=G(\tilde{F}, \tilde{\mathcal{X}}) \rightarrow G=G(F, \mathcal{X})
$$

is a covering space projection.

Since each type (B) cover is always done just after a type (A) cover, it follows from Theorem 2.12 and Lemma 2.14 that $\sigma$ is injective but not surjective so $\widetilde{G}$ consists of $2^{k}$ disjoint copies of the universal $\mathbb{Z} / 2$-cover of $G$ with $k>0$. Hence the number of vertices (and hence $|\partial F|$ ) and $e(F)$ are all multiplied by $2^{m}$, where $m=$ $k+\beta_{1}(G(\mathcal{S}))>0$. Thus spider covers of type (B) preserves properties (F2), (F3).

We assert that $\ell(\widetilde{G})=2 \ell(G)$. Suppose $\alpha$ is an essential loop in $\widetilde{G}$ of minimal length. Then it is a simple closed curve. It projects to an essential loop $\beta$ in $G$ which crosses 
each edge of $G$ an even number of times because it lifts to the loop $\alpha$. Hence the restriction of $p_{G}$ to $\alpha$ is a 2 -fold covering of $\beta$, which proves the assertion.

The initial graph is bipartite so initially $\ell \geq 2$. After doing a type (B) cover twice, $\ell \geq 8$ and $|\partial F| \geq 4$ and $e(F)$ is even. The final type (A) cover restores (F1). This proves the claim.

We replace the original spider surface by one with the above properties and show that if $e>0$ then there is a spider cover that reduces $e$ by 2 and continues to have these properties. Continuing reduces $e$ to 0 , which is a spider surface that spider-covers the original and has properties (F1) and (F2), proving the theorem.

If $e \neq 0$, then $e \geq 2$ by (F3) and there are two cases to consider:

Case 1 There are components $C \neq C^{\prime}$ of $\partial F$ with $e(C) \geq 1$ and $e\left(C^{\prime}\right) \geq 1$.

Case 2 There is a component $C$ of $\partial F$ with $e(C) \geq 2$.

Below we describe two spider covers of spider degree 1 that reduce $e$ by 2 and increase $|\partial F|$ by 2 , thus they preserve (F2) and (F3). They preserve (F4) by Lemma 2.15(G2). In both cases we follow the cover by a type (A) cover. The latter restores (F1), and gives an isomorphic spider graph, so it does not change $e$, and preserves (F2)-(F4).

Case 1 Using (F1) and (F2) there is a 2-fold cover $p: \widetilde{F} \rightarrow F$ such that every spider in $\mathcal{X}$ lifts and $C$ and $C^{\prime}$ are the only components of $\partial F$ with two disjoint lifts.

To construct this cover: by (F2) and (F1) we may choose a finite number of pairwise disjoint properly embedded arcs in $F$ that are disjoint from $\mathcal{X}$, whose union has exactly one endpoint on each component of $\partial F$ except $C$ and $C^{\prime}$. There is at least one such arc by (F2), so these arcs represent a nontrivial element of $H^{1}(F ; \mathbb{Z} / 2)$ and determine $p$. In fact cross-joining two copies of $F$ along this family of arcs gives the cover.

To construct a spider cover it remains to choose one lift of each spider to obtain $\tilde{\mathcal{X}} \subset \tilde{F}$. This must be done so $\tilde{\mathcal{X}}$ has ample spiders (S3). Then replacing $F$ with $\tilde{F}$ increases the number of boundary components of $F$ by 2 without changing the number of spider feet, so this reduces the excess by 2 .

By (F4) no spider has two feet on $C$ so there are at least two distinct spiders $X_{1}$ and $X_{2}$ both with feet on $C$. Similarly there are $X_{1}^{\prime}$ and $X_{2}^{\prime}$ for $C^{\prime}$. It is possible some $X_{i}$ equals some $X_{j}^{\prime}$. If this is the case we label so that $X_{1}=X_{1}^{\prime}$. However $X_{2} \neq X_{2}^{\prime}$ because $G(\mathcal{S})$ contains no circuit of length 4 .

Since the covering is regular, at least one of the two lifts of $X_{i}$ has a spider foot on a given lift of $C$. Choose lifts of $X_{1}$ and $X_{2}$ so that both of the boundary components 
covering $C$ contain a spider foot. If $X_{1} \neq X_{1}^{\prime}$, choose an arbitrary lift of $X_{1}^{\prime}$. It is possible that the lift of $X_{1}^{\prime}$ has spider feet on both lifts of $C^{\prime}$. In this case choose any lift of $X_{2}^{\prime}$. Otherwise, since $X_{2} \neq X_{2}^{\prime}$, we are free to choose a lift of $X_{2}^{\prime}$ that has a spider foot on the lift of $C^{\prime}$ that does not contain a spider foot on the chosen lift of $X_{1}$. The remaining spiders may be lifted in any way. This ensures the lifted spiders are ample (S3).

Case 2 There is a 3 -fold cyclic cover $p: \widetilde{F} \rightarrow F$ such that every spider in $\mathcal{X}$ lifts, the only component of $\partial F$ with more than one pre-image is $C$, and $C$ has 3 pre-images.

To construct this cover, since $|\partial F| \geq 4$ and is even, there is a finite set of pairwise disjoint arcs properly embedded in $F$, so $C$ contains one endpoint of each of exactly 3 distinct arcs and every other component of $\partial F$ contains one arc endpoint. By (F1) we may choose these arcs disjoint from $\mathcal{X}$. Choose a transverse orientation on these arcs so that the arcs that meet $C$ induce the same orientation on $C$. These transversally oriented arcs represent an element of $H^{1}(F ; \mathbb{Z} / 3)$ and determine $p$. As before, the cover can be constructed by cyclically cross-joining 3 copies of $F$ along these arcs.

By (F4) there are at least 3 distinct spiders with feet on $C$. Choose lifts of these so that there is at least one spider foot on each pre-image of $C$. The remaining spiders can be lifted in any way and the result is ample (S3). As before, replacing $F$ by $\widetilde{F}$ reduces the excess by 2 .

\section{The intersection of quasi-Fuchsian manifolds}

Suppose $Q_{1}$ and $Q_{2}$ are QF 3-manifolds embedded in a hyperbolic 3-manifold $M$. We assume the rank-1 cusps of $Q_{1}$ and $Q_{2}$ have different slopes in each rank-2 cusp of $M$. Then each component $R$ of $Q_{1} \cap Q_{2}$ is called an ideal 3-spider Definition 3.2 and is the union of a compact, convex manifold $R^{c}$ and finitely many ends called legs; see Proposition 3.3. In Theorem 3.4 we generalize this to when $Q_{i}$ are immersed in $M$ rather than embedded. This gives an immersed ideal 3-spider $R \rightarrow Q_{i}$.

Next, Proposition 3.5 gives a two-dimensional approximation of this immersion by an immersion $X \rightarrow F_{i}$, where $F_{i}$ is a finite-area hyperbolic surface with cusps and $X$ a convex surface called an ideal 2-spider. An ideal 2-spider is the union of a compact convex part and finitely many ends called legs, each of which maps to a regular neighborhood of a ray going out into a cusp of $F_{i}$.

Truncating the cusps of $F_{i}$, cutting the legs off the ideal spider $X$, and changing the metric gives a (compact) immersed spider as defined in Section 2. Finally Corollary 3.10 shows how the problem of finding covers of QF 3-manifolds with gluing regions 
that are far apart and with simple combinatorics is related to the spider theorem. In Theorem 3.13 we relate spiders to some earlier work of Anderson and Soma.

Suppose $\mathcal{B} \subset \mathbb{H}^{n}$ is a horoball centered on a point $x \in \partial \mathbb{H}^{n}$ bounded by the horosphere $\mathcal{H}=\partial \mathcal{B}$. A vertical ray is a ray in $\mathcal{B}$ that starts on $\mathcal{H}$ and limits on $x$. Given $P \subset \mathcal{H}$, the set lying above $P$ is called a vertical set and is the union $V(P)$ of the vertical rays starting on $P$. If $P$ is convex, $V(P)$ is called a thorn and $P$ is called the base of the thorn. A thorn of dimension 2 is also called a spike. If $P=I \times \mathbb{R}$ is an infinite strip, $V(P)$ is a slab.

A hyperbolic $n$-manifold $E$ is an excellent end if it has finite volume and is isometric to $V / \Gamma$ for some vertical set $V \subset \mathcal{B}$ and discrete group $\Gamma \subset \operatorname{Isom}\left(\mathbb{H}^{n}\right)$ preserving $V$. The horospherical boundary of $E$ is $\partial_{\mathcal{H}} E=(V \cap \mathcal{H}) / \Gamma$. An excellent rank-1 cusp is a 3 -manifold $V / \Gamma$, where $V$ is a slab and $\Gamma$ is a cyclic group of parabolics preserving $V$.

A (possibly not connected) hyperbolic manifold $M$ is excellent if $M=M^{c} \cup \mathcal{V}_{M}$, where $M^{c}$ is compact and $M^{c} \cap \mathcal{V}_{M}=\partial_{\mathcal{H}} \mathcal{V}_{M}$ and each component of $\mathcal{V}_{M}$ is an excellent end. The pair $\left(M^{c}, \mathcal{V}_{M}\right)$ is called an excellent decomposition of $M$. For example, an ideal convex polytope is excellent and the ends are thorns. Also, a complete hyperbolic $n$-manifold with finite volume is excellent since the ends are horocusps. Observe that an excellent manifold has finite volume.

If $N$ is a hyperbolic 3-manifold and $S \subset N$ is an incompressible surface with holonomy $\Gamma$, then $S$ is a $Q F$ surface if $M_{S}=\mathbb{H}^{3} / \Gamma$ is QF. The convex core of $M_{S}$ is a 3-manifold unless $S$ is Fuchsian, in which case it is $S$. To overcome this mild technical irritation we define a convex 3-manifold by $Q(S)=\operatorname{Core}\left(M_{S}\right)$ unless $S$ is Fuchsian, in which case $Q(S)=\mathrm{CH}(S \cup U)$, where $U \subset M_{S}$ is a small open set that meets $S$. It is routine to show that if $S$ is a QF surface then $Q(S)$ has ends that are excellent rank-1 cusps, thus $Q(S)$ is excellent.

A compact, orientable surface properly embedded in a compact orientable 3-manifold is essential if it is incompressible and $\partial$-incompressible.

Definition 3.1 A surface $S$ embedded in an excellent 3-manifold $M=M^{c} \cup \mathcal{V}$ is excellently essential if each component of $S \cap \mathcal{V}$ is an excellent annulus, and $S^{c}=S \cap M^{c}$ is a compact essential surface in $M^{c}$ with $\partial S^{c} \subset M^{c}$.

A slope on a torus is an isotopy class of essential simple closed curves. In view of the preceding, it makes sense to talk about the slope of a excellently essential surface $S$ in a cusp of $M$, and the slope of a rank- 1 cusp embedded in a rank-2 cusp. 
Definition 3.2 An ideal $n$-spider is an excellent convex hyperbolic $n$-manifold $X$ with simply connected ends. Thus there is an excellent decomposition $X=B \cup \mathcal{L}$ such that $B$ is compact and convex and each component of $\mathcal{L}$ is a thorn. The components of $\mathcal{L}$ are called legs and $B$ is called the body.

If the dimension $n$ is clear from context we will omit it and talk about an ideal spider. The definition implies that the holonomy of an ideal spider has no parabolics. A convex ideal polytope with $k$ ideal vertices is an ideal spider with $k$ legs. An ideal spider is degenerate if it is a bounded regular neighborhood of a geodesic. The following is obvious:

Proposition 3.3 Suppose $M$ is a complete hyperbolic 3-manifold with finite volume and $Q_{1}, Q_{2} \subset M$ are excellent $Q F$ submanifolds. Then $Q_{1} \cap Q_{2}$ is excellent. If $Q_{1}$ and $Q_{2}$ have different slopes in every cusp of $M$, then each component of $Q_{1} \cap Q_{2}$ is an ideal spider.

If $M$ and $N$ are excellent hyperbolic manifolds a map $f: M \rightarrow N$ is excellent if it is a local isometry and there are excellent decompositions with $f^{-1}\left(N^{c}\right)=M^{c}$. It follows that each vertical ray in $\mathcal{V}_{M}$ maps to a vertical ray in $\mathcal{V}_{N}$.

An immersed $Q F$ manifold is $(M, Q, f)$ where $f: Q \rightarrow M$ is an excellent map between excellent hyperbolic 3-manifolds and $Q$ is QF. Two immersed QF manifolds $\left(M, Q_{1}, f_{1}\right)$ and $\left(M, Q_{2}, f_{2}\right)$ have different slopes if for every cusp $V_{i} \subset Q_{i}$, whenever $f_{1}\left(V_{1}\right)$ and $f_{2}\left(V_{2}\right)$ are in the same cusp of $M$ they have different slopes. An immersed ideal $n$-spider is $(M, R, p)$ where $M$ is an excellent $n$-manifold and $R$ is an ideal $n$-spider and $p: R \rightarrow M$ is excellent.

Suppose $Q$ is an excellent QF 3-manifold and $(Q, R, p)$ is an immersed ideal 3spider. We show in Proposition 3.5 that this is approximated by an immersed ideal 2 -spider $(F, X, f)$ for some complete hyperbolic surface $F$ with cusps.

If $N$ is a submanifold of a covering of a hyperbolic manifold $M$, the restriction of the covering space projection gives a local isometry $p: N \rightarrow M$ called the natural projection. If $S$ is a QF surface in $M$ it is easy to see that the natural projection $Q(S) \rightarrow M$ is excellent. The following generalizes Proposition 3.3 to immersed $\mathrm{QF}$ manifolds.

Theorem 3.4 Suppose that $\left(M, Q_{1}, f_{1}\right)$ and $\left(M, Q_{2}, f_{2}\right)$ are two immersed $Q F$ manifolds with different slopes. Suppose $q_{i} \in Q_{i}$ and the basepoint $m=f_{1}\left(q_{1}\right)=$ $f_{2}\left(q_{2}\right)$ is in a horocusp of $M$. 
Then there is a connected hyperbolic 3-manifold $P=\widetilde{Q}_{1} \cup \widetilde{Q}_{2}$, where $p_{i}: \widetilde{Q}_{i} \rightarrow Q_{i}$ is a finite covering and $R=\widetilde{Q}_{1} \cap \widetilde{Q}_{2}$ is an ideal 3-spider with at least 2 legs, thus $\left(Q_{i}, R,\left.p_{i}\right|_{R}\right)$ is an immersed ideal spider.

The holonomy provides an identification of $\pi_{1}(M, m)$ with a Kleinian group $\Gamma \subset$ Isom $\left(\mathbb{H}^{3}\right)$. Then the $Q F$ manifolds $Q_{i}$ have holonomy $\Gamma_{i}=\left(f_{i}\right)_{*}\left(\pi_{1}\left(Q_{i}, q_{i}\right)\right) \subset \Gamma$ and the holonomy of $R$ is $\Gamma_{1} \cap \Gamma_{2}$.

Proof This is a special case of the virtual simple gluing theorem (4.3) in [2]. For the convenience of the reader we include a self-contained proof.

Let $Q_{i}^{\prime} \subset \mathbb{H}^{3}$ be the embedding of the universal cover of $Q_{i}$ preserved by $\Gamma_{i}$ so $Q_{i}=Q_{i}^{\prime} / \Gamma_{i}$. The set $R^{\prime}=Q_{1}^{\prime} \cap Q_{2}^{\prime}$ is convex hence so is the manifold $R=R^{\prime} / \Gamma_{R}$, where $\Gamma_{R}=\operatorname{Stab}\left(R^{\prime}\right)=\Gamma_{1} \cap \Gamma_{2}$. We prove $\Gamma_{R}$ is finitely generated. Hence it is a separable subgroup of the free group $\Gamma_{i}$. Since $R$ is convex it embeds in some finite covers of the $Q_{i}$. These coverings are then glued to produce $P$ by identifying the two copies of $R$.

We first prove the corresponding statements for the compact cores obtained by removing the cusps and then deduce the result by gluing the cusps back on and using the fact they are excellent.

There are excellent decompositions with compact submanifolds $Q_{i}^{c} \subset Q_{i}$ and $M^{c} \subset M$ with $m$ outside $M^{c}$ and $f_{i}^{-1} M^{c}=Q_{i}^{c}$. There is a natural projection $p_{R}: R \rightarrow M$ and we define $R^{c}=p_{R}^{-1} M^{c}$. The pre-image $Y \subset R^{\prime}$ of $R^{c}$ is obtained from $R^{\prime}$ by removing the intersections with the interiors of the pairwise disjoint horoballs covering cusps in $M$. It follows that $Y$, and hence $R^{c}$, are connected. If $U$ is a subset of a convex hyperbolic manifold $Z$, define $N_{1}(U)=\mathcal{N}_{1}\left(U, T h_{1}(Z)\right)$. We first show that $R^{c}$ is compact by showing that $\operatorname{vol}\left(N_{1}\left(R^{c}\right)\right)<\infty$. Observe that since $Q_{i}^{c}$ is compact $\operatorname{vol}\left(N_{r}\left(Q_{i}^{c}\right)\right)<\infty$ for all $r \geq 0$.

There are natural projections $g_{i}: R \rightarrow Q_{i}$ with $f_{1} \circ g_{1}=f_{2} \circ g_{2}$. If $\operatorname{vol}\left(N_{1}\left(R^{c}\right)\right)=\infty$ then, since $\operatorname{vol}\left(N_{1}\left(Q_{1}^{c}\right)\right)<\infty$, there is a point $a \in N_{1}\left(Q_{1}^{c}\right)$ with infinitely many preimages $\mathcal{A}=g_{1}^{-1}(a)$ in $N_{1}\left(R^{c}\right)$. These project to the same point in $M$, hence there is $0<\delta<1$ such that the $\delta$-balls in $N_{2}\left(R^{c}\right)$ centered on the points of $\mathcal{A}$ are all pairwise disjoint.

The pre-image $\tilde{\mathcal{A}} \subset N_{1}\left(R^{\prime}\right)$ of $\mathcal{A}$ is contained in finitely many $\Gamma_{i}$-orbits; otherwise $N_{2}\left(Q_{i}^{c}\right)$ contains infinitely many pairwise disjoint $\delta$-balls, contradicting it has finite volume. Hence at least one of the orbits $\Gamma_{1} \cdot \tilde{a}$ is infinite. But this orbit is contained in $N_{1}(Y) \subset N_{1}\left(\left(Q_{2}^{c}\right)^{\prime}\right)$ and since $Q_{2}^{c}$ is compact, the set $\Gamma_{1} \cdot \tilde{a}$ is contained in finitely many $\Gamma_{2}$-orbits. Hence there are two distinct points of $\mathcal{A}$ with pre-images in $\tilde{\mathcal{A}}$ that 
are in the same $\Gamma_{R}=\Gamma_{1} \cap \Gamma_{2}$ orbit. But this means they have the same image in $\mathcal{A} \subset R=\widetilde{R} / \Gamma_{R}$, a contradiction to the assumption that $|\mathcal{A}|=\infty$. This proves the claim.

Since $R^{c}$ is compact it follows that $\pi_{1} R=\Gamma_{R}$ is finitely generated. The 3 -manifold $\left(Q_{1}^{\prime} \cup Q_{2}^{\prime}\right) / \Gamma_{R}$ contains $R$ as a submanifold. Using subgroup separability in the free groups $\Gamma_{i}$, there are finite-index subgroups $\Gamma_{i}^{\prime} \subset \Gamma_{i}$ giving finite covers $p_{i}: \widetilde{Q}_{i} \rightarrow Q_{i}$ and lifts $\widetilde{g}_{i}: R \rightarrow \widetilde{Q}_{i}$ with $p_{i} \circ \tilde{g}_{i}=g_{i}$ and $\widetilde{g}_{i} \mid R^{c}$ injective.

A hyperbolic 3-manifold $P$ is obtained from $Q_{1}^{\prime} \cup Q_{2}^{\prime} \subset \mathbb{H}^{3}$ by using $\Gamma_{i}^{\prime}$ to identity points in $Q_{i}^{\prime}$. Let $P^{c}$ be the submanifold that is the pre-image of $M^{c}$ under the natural projection. Since $Q_{i}$ is excellent so is $\widetilde{Q}_{i}$ and thus so is $P$. Since the ends of $P$ are vertical it follows that $g_{i}$ is injective on all of $R$. Any identifications in the ends of $R$ would produce identifications on $\partial R^{c}$ because the ends are excellent.

The hypothesis that the cusps of $Q_{1}=Q\left(S_{1}\right)$ and $Q_{2}=Q\left(S_{2}\right)$ always have different slopes implies the ends of $R$ are thorns. The spider $R$ has at least 2 legs because the basepoint $m$ is in a cusp of $M$, so $R$ contains an essential arc in $S_{1} \cap S_{2}$ that contributes two legs.

The manifold $R$ produced by this theorem is called a gluing region and the manifold $P$ is called the manifold obtained by gluing $\widetilde{Q}_{1}$ to $\widetilde{Q}_{2}$ along $R$. In general $P$ does not have a convex thickening.

The Hausdorff distance $\delta(A, B)=\delta_{X}(A, B)$ between two closed subsets $A, B \subset X$ of a metric space $X$ is the infimum of $K \in[0, \infty]$ such that $A$ is contained in a $K$-neighborhood of $B$, and $B$ is contained in a $K$-neighborhood of $A$.

The next result provides an immersed ideal 2-spider $(F, X, g)$ that approximates an immersed ideal 3-spider $(Q(S), R, p)$ in the sense that there is a bilipschitz homeomorphism between universal covers of $Q(F)$ and $Q(S)$ taking each pre-image of $X$ close (in the sense of Hausdorff distance) to a pre-image of $R$.

Proposition 3.5 Suppose $Q=Q(S)$ is $Q F$ and $(Q, R, p)$ is an immersed ideal 3spider with $k \geq 2$ legs. Then there is an immersed ideal $2-$ spider $(F, X, f)$ with $k$ legs that approximates it in the following sense. There is a bilipschitz homeomorphism $h: Q \rightarrow \underset{\widetilde{f}}{Q(F)}$ such that if $\widetilde{R}, \tilde{X}, \widetilde{Q}$ are universal covers and $\tilde{p}: \widetilde{R} \rightarrow \widetilde{Q}$ covers $p$, there is $\widetilde{f}: \tilde{X} \rightarrow \widetilde{F}$ covering $f$ such that

$$
\delta\left(\tilde{p}(\tilde{R}), \tilde{h}^{-1} \circ \tilde{f}(\tilde{X})\right)<\infty .
$$

Here we regard $F$ as a flat surface in $Q(F)$. Moreover, $f_{*}\left(\pi_{1} X\right)=p_{*}\left(\pi_{1} R\right)$. 
Proof By Theorem 3.12 there is a quasiconformal automorphism $H$ of $\overline{\mathbb{H}}^{3}$ that conjugates the holonomy $\Gamma_{S}$ of $Q$ to the holonomy $\Gamma_{F}$ of $F$ and there is a bilipschitz homeomorphism $h$ as required and $\tilde{h}=H \mid \mathbb{H}^{3}$. We identify the universal cover $\widetilde{Q}$ with a subset of $\mathbb{H}^{3}$ and identify $\widetilde{R}$ with $\widetilde{p}(\widetilde{R}) \subset \mathbb{H}^{3}$.

Define $Z=\operatorname{CH}(\Lambda(\widetilde{R}))$ and $\tilde{X}=\mathrm{CH}(H(\Lambda(\widetilde{R})))$. Now $\Lambda(\widetilde{R}) \subset \Lambda(\widetilde{Q})$ and

$$
H(\Lambda(\widetilde{Q}))=\Lambda(\widetilde{F})=\partial \mathbb{H}^{2} \subset \partial \mathbb{H}^{3} .
$$

Thus $H(\Lambda(\widetilde{R})) \subset \partial \mathbb{H}^{2}$, hence $\tilde{X} \subset \mathbb{H}^{2}$.

Let $\Gamma_{\widetilde{R}} \subset \Gamma$ be the stabilizer of $\widetilde{R}$, so $\Gamma_{\widetilde{R}} \cong \pi_{1} R$. Then $\Gamma_{\tilde{X}}=H\left(\Gamma_{\widetilde{R}}\right) H^{-1}$ preserves $\tilde{X}$ and we obtain a hyperbolic surface $X=\tilde{X} / \Gamma_{\tilde{X}}$. Since $\Gamma_{\tilde{X}} \subset \Gamma_{F}$ there is natural projection $f: X \rightarrow F$.

This identification of $\tilde{X}$ with a subset of $\mathbb{H}^{2} \subset \mathbb{H}^{3}$ makes $\tilde{f}$ the inclusion map, and we omit it in what follows, so that $\tilde{X}=\tilde{f}(\tilde{X})$. With these identifications, we must show $\delta\left(\tilde{R}, \tilde{h}^{-1}(\tilde{X})\right)<\infty$. This follows from the next two claims.

Claim $1 \delta(\widetilde{R}, Z)<\infty$.

Since $R$ is convex, $Z \subset \widetilde{R}$, so it suffices to show there is an upper bound on the distance of points in $\widetilde{R}$ from $Z$. There is an upper bound on the distance of a point in a thorn from a geodesic ray running down the thorn. Since $R=B \cup \mathcal{L}$ is the union of a compact submanifold $B$ (the body of the spider) and $k \geq 2$ legs (which are thorns) there is $K>0$ such that every point $x \in R$ is within distance $K$ of some bi-infinite geodesic $\gamma=\gamma(x)$ in $R$ that starts in one leg of $R$ and ends in another. These geodesics lift into $Z$, proving the claim.

Claim $2 \delta\left(Z, \tilde{h}^{-1}(\tilde{X})\right)<\infty$.

By Lemma 3.11 every point in $Z$ is distance less than 2 from a geodesic $\gamma$ with endpoints in $\Lambda(\widetilde{R})=\Lambda(Z)$. Since $\tilde{h}$ is bi-Lipschitz, $\tilde{h}(\gamma)$ is a quasi-geodesic, so there is $K>0$ independent of $\gamma$ such that $\tilde{h}(\gamma)$ lies within a distance $K$ of a geodesic $\gamma^{\prime}$ with endpoints in $\tilde{h}(\Lambda Z)$. Thus $\gamma^{\prime} \subset \tilde{X}$, hence $\delta(\tilde{h}(Z), \tilde{X})<\infty$. Since $H$ is bilipschitz, $\delta\left(Z, \tilde{h}^{-1}(\tilde{X})\right)<\infty$.

If $R$ is a degenerate ideal 3 -spider, it is easy to see that $\tilde{X}$ is a bi-infinite geodesic. In this case we thicken $\tilde{X}$ slightly in $\mathbb{H}^{2}$ to get a degenerate ideal 2 -spider.

Claim $3(F, X, f)$ is an immersed ideal 2-spider with $k$ legs. 
If $R$ is simply connected then, except in the degenerate case above, $\tilde{X} \cong X$ is an ideal polygon, hence an ideal spider, with $k=|\Lambda(\widetilde{R})|$ vertices. In general we establish a similar picture in the covers $Q^{\prime}$ of $Q$ and $Q^{\prime}(F)$ of $Q(F)$ corresponding to $\pi_{1} R$.

Let $h^{\prime}: Q^{\prime} \rightarrow Q^{\prime}(F)$ be the map covered by $\tilde{h}$. The projections of $\widetilde{R}$ and $\tilde{X}$ give submanifolds $R^{\prime} \subset Q^{\prime}$ and $X^{\prime} \subset Q^{\prime}(F)$ homeomorphic to $R$ and $X$ that are the images of lifts of $p$ and $f$.

Since $R$ and $Q^{\prime}$ are convex and have the same fundamental group $Q^{\prime}$ is a convex thickening of $R^{\prime}$. Similarly $Q^{\prime}(F)$ is a convex thickening of $X^{\prime}$. Since $R^{\prime}$ is a lift of $R$ it follows that $R^{\prime}=B^{\prime} \cup \mathcal{L}^{\prime}$ is the union of a compact body $B^{\prime}$, and $k$ legs. There is a geodesic in $R^{\prime}$ running from any leg to any other leg. Since $Q^{\prime}$ is a convex thickening of $R^{\prime}$ this geodesic is distance minimizing between any pair of points on it. Thus the distance between distinct legs of $R^{\prime}$ goes to infinity outside compact sets. Since $h^{\prime}$ is bilipschitz the image of a leg of $R^{\prime}$ is contained in some $K$-neighborhood of a geodesic ray in $Q^{\prime}(F)$. Now $X^{\prime}$ is a convex surface and $\delta\left(h^{\prime}\left(R^{\prime}\right), X^{\prime}\right)<\infty$, so $X^{\prime}$ has a leg (spike) close to the image of each leg of $R^{\prime}$. Thus $X$ is the union of a compact subsurface and $k$ spikes, hence a 2 -spider.

Informally, a wall is obtained from a QF manifold with finitely many immersed ideal 3 -spiders by deleting the cusps.

\section{Definition 3.6 Suppose:}

(W1) $Q=Q^{c} \cup \mathcal{V}$ is an excellent QF manifold.

(W2) $\mathcal{R}$ is the disjoint union finitely many ideal 3 -spiders and $p: \mathcal{R} \rightarrow Q$ is excellent.

(W3) $(Q, R, p \mid R)$ is an immersed ideal 3-spider for each component $R \subset \mathcal{R}$.

(W4) (Ample spiders) $p^{-1}(V) \neq \phi$ for each component $V \subset \mathcal{V}$.

Components of $\mathcal{R}^{c}:=p^{-1} Q^{c}$ are called gluing regions and $W=\left(Q^{c}, \mathcal{R}^{c}, p \mid: \mathcal{R}^{c} \rightarrow\right.$ $\left.Q^{c}\right)$ is called a wall. The base of the wall is $\partial_{b} Q^{c}=Q^{c} \cap \mathcal{V}$. A wall is simple if $p^{-1}(V)$ is connected for each component $V \subset \mathcal{V}$. This means each component of the base of the wall intersects exactly one gluing region.

Definition 3.7 Suppose $W=\left(Q^{c}, \mathcal{R}^{c}, p\right)$ and $\widetilde{W}=\left(\widetilde{Q}^{c}, \widetilde{\mathcal{R}}^{c}, \widetilde{p}\right)$ are walls. A wall cover $\left(\pi, \pi^{\prime}\right): \widetilde{W} \rightarrow W$ with gluing degree $d$ consists of covering space maps $\pi: \widetilde{Q}^{c} \rightarrow Q^{c}$ and $\pi^{\prime}: \widetilde{\mathcal{R}}^{c} \rightarrow \mathcal{R}^{c}$ such that $\widetilde{\mathcal{R}}^{c}$ is the disjoint union of $d$ copies of $\mathcal{R}^{c}$ and $\pi^{\prime}$ is the natural projection, and the following diagram commutes:




We will show every wall is covered by a simple wall with embedded gluing regions.

Definition 3.8 A connected spider surface $\mathcal{S}=(F, \mathcal{X}, f)$ approximates a wall $W=$ $\left(Q^{c}, \mathcal{R}^{c}, p\right)$ if there is a diffeomorphism $h: I \times F \rightarrow Q^{c}$ with $h(I \times \partial F)=Q^{c} \cap \mathcal{V}$ and the following holds. Let $\widetilde{F}$ and $\widetilde{Q}^{c}$ be the universal covers and $\widetilde{h}: I \times \widetilde{F} \rightarrow \widetilde{Q}^{c}$ cover $h$. Let $A$ be the set of all submanifolds $I \times \tilde{f}(\tilde{X}) \subset I \times \tilde{F}$, where $\tilde{X}$ is the universal cover of a component $X \subset \mathcal{X}$ and $\tilde{f}$ covers $\left.f\right|_{X}: X \rightarrow F$. The action of $\pi_{1} F$ on $\widetilde{F}$ induces an action on $A$. Let $B$ denote the set of submanifolds of $\widetilde{p}\left(\widetilde{R}^{c}\right) \subset \widetilde{Q}^{c}$, where $\widetilde{R}$ is the universal cover of a component $R \subset \mathcal{R}^{c}$ and $\tilde{p}$ covers $p: R \rightarrow Q$. The action of $\pi_{1} Q^{c}$ on $\widetilde{Q}^{c}$ induces an action on $B$.

We require there is $K>0$ and a bijection $\theta: A \rightarrow B$ such that $\delta(x, \theta(x))<K$. This bijection is equivariant for the actions of $\pi_{1} F$ and $\pi_{1} Q$ via $h_{*}$. We also require that for every component $C \subset I \times \partial \tilde{F}$ and $x \in A$ that $x \cap C \neq \phi$ if and only if $\theta(x) \cap \tilde{h}(C) \neq \phi$.

It is routine to check that if $\mathcal{S}$ approximates $W$ then a spider cover $\widetilde{\mathcal{S}}$ induces a wall cover $\widetilde{W}$ and $\widetilde{\mathcal{S}}$ approximates $\widetilde{W}$. Moreover $W$ is simple if and only if $\mathcal{S}$ is simple.

Corollary 3.9 Every wall is approximated by an immersed spider surface.

Proof By Proposition 3.5 an immersed ideal 3-spider $(Q, R, p)$ is approximated by an immersed ideal 2-spider $(F, X, f)$. There are excellent decompositions $F=$ $F^{c} \cup \mathcal{V}_{F}$ and $X=X^{c} \cup \mathcal{V}_{X}$ where each component of $\mathcal{V}_{F}$ is a cusp and each component of $\mathcal{V}_{X}$ is a spike and $f$ is excellent for this decomposition. There is a hyperbolic metric on $F^{c}$ so that $F^{c}$ has geodesic boundary, and the pullback to $X^{c}$ using $f$ makes $X$ convex. With these new metrics $\left(F^{c}, X^{c}, f \mid\right)$ is an immersed spider. It is routine to check the conclusion follows from Proposition 3.5.

An excellent convex $n$-manifold $M=M^{c} \cup \mathcal{V}$ is a submanifold of the complete manifold $M^{\infty}=\mathrm{Th}_{\infty}(M)$. Each component $V \subset \mathcal{V}$ is covered by a vertical subset of some horoball $\mathcal{B} \subset \mathbb{H}^{n}$. The image of $\mathcal{B}$ in $M^{\infty}$ is a vertical submanifold $V^{\infty}$ that is a thickening of $V$. Let $\mathcal{V}^{\infty} \subset M^{\infty}$ be the union of all such; then it is a thickening of $\mathcal{V}$ and is the quotient by $\pi_{1} M$ of a collection of pairwise disjoint horoballs. Given $\kappa \geq 0$ the relative $\kappa$-thickening $\operatorname{Th}_{\kappa}^{\text {rel }}(M)$ of $M$ is the convex hull of $X_{\kappa}(M)=\operatorname{Th}_{k}(M) \backslash \mathcal{V}^{\infty}$. It is the union of $X_{\kappa}(M)$ and excellent ends in $\mathcal{V}^{\infty}$ that lie vertically above $\operatorname{Th}_{\kappa}(M) \cap \partial \mathcal{V}^{\infty}$. It is a thickening of $M$ and it is excellent. It contains a $\kappa$-neighborhood of $M^{c}$. If $W=\left(Q^{c}, \mathcal{R}^{c}, p\right)$ is a wall and $\kappa>0$ then the $\kappa$-thickened wall $W^{\kappa}$ consists of the relative $\kappa$ thickenings of $Q$ and $\mathcal{R}$ truncated along the same cusps. 
The following allows us to reformulate the problem of finding a simple cover of a wall with lifts of gluing regions that are far apart as a corollary of the spider theorem.

Corollary 3.10 Suppose that $W$ is a wall and $\mathcal{S}$ is a spider surface that approximates $W$. Given $\kappa>0$ there is $\delta>0$ such that if $\widetilde{\mathcal{S}}^{\delta}$ is a simple embedded spider cover of $\mathcal{S}^{\delta}$, then the corresponding cover $\widetilde{W}^{\kappa}$ is simple and the gluing regions are pairwise disjoint.

Proof Refer to Proposition 3.5. If $\delta$ is sufficiently large then the corresponding wall cover $\widetilde{W}^{\kappa}$ has embedded gluing regions. This is because if $R$ is an ideal spider then $\mathrm{Th}_{\kappa}^{\text {rel }}(R)$ is convex so its universal cover embeds isometrically in $\mathbb{H}^{3}$. The argument in the proof of Theorem 3.4 implies if $\delta$ is large enough then the lifts of $X_{\kappa}(R)$ to $\widetilde{W}$ corresponding to the lifted spiders in $\widetilde{\mathcal{S}}^{\delta}$ are pairwise disjoint. The map $h$ is covered by a $K$-bilipschitz homeomorphism between $\widetilde{\mathcal{S}}^{\delta}$ and $\widetilde{W}^{\kappa}$. Hence for $\delta$ large the lifted gluing regions in $\widetilde{W}$ are far apart.

Lemma 3.11 If $R \subset \overline{\mathbb{H}}^{3}$ is any subset, then every point in $\mathrm{CH}(R)$ is within a distance 2 of a geodesic segment with endpoints in $R$.

Proof This easily follows from the fact that the convex hull of $R$ is the union of the (ideal) simplices with vertices in $R$, and the thin triangles constant for hyperbolic space is less than 1 .

Theorem 3.12 Suppose $S$ is a $Q F$ surface, so $Q(S)$ is a finite-volume convex submanifold of the complete $Q F$ manifold $M_{S}$. Suppose $F$ is a finite-area hyperbolic surface that is homeomorphic to $S$.

Then there is a bilipschitz homeomorphism $h: M_{S} \rightarrow M_{F}$ with $h(Q(S))=Q(F)$ and a quasi-conformal automorphism $H$ of $\overline{\mathbb{H}}^{3}$ such that $H \mid \mathbb{H}^{3}$ covers $h$.

Proof This is well known except for the fact we may arrange $h(Q(S))=Q(F)$. This follows from the fact there is a bilipschitz self-homeomorphism of $M_{F}$ that takes $Q(F)$ to $h(Q(S))$. This uses that $Q(F)$ and $Q(S)$ are the union of a diffeomorphic compact part and excellent cusps.

\section{Spiders in (relation to) the work of Anderson and Soma}

We do not make use of the following. Suppose $\Gamma_{1}, \Gamma_{2}$ are QF subgroups of a Kleinian group $\Gamma$ and that $x \in \partial \mathbb{H}^{3}$ is fixed by non-trivial parabolics $\gamma_{1} \in \Gamma_{1}$ and $\gamma_{2} \in \Gamma_{2}$. The subgroup $\Gamma^{\prime}$ of $\Gamma$ generated by $\gamma_{1}$ and $\gamma_{2}$ is discrete and is free abelian of rank 1 or 2 . If $\Gamma^{\prime}$ has rank 2 then $\gamma_{1}$ and $\gamma_{2}$ translate in different directions in a horosphere 
$\mathcal{H} \subset \mathbb{H}^{3}$ centered at $x$ and represent different slopes on the quotient horotorus $\mathcal{H} / \Gamma^{\prime}$. Let $P\left(\Gamma_{1}, \Gamma_{2}\right) \subset \partial \mathbb{H}^{3}$ denote the (possibly empty) set of all such points. Anderson calls this the exceptional set.

The following is an immediate consequence of Anderson's [1, Theorem C]; see also Soma [13].

Theorem 3.13 Suppose $\Gamma_{1}, \Gamma_{2}$ are $Q F$ subgroups of a Kleinian group $\Gamma$. Then $\Lambda\left(\Gamma_{1}\right) \cap \Lambda\left(\Gamma_{2}\right)=\Lambda\left(\Gamma_{1} \cap \Gamma_{2}\right) \cup P\left(\Gamma_{1}, \Gamma_{2}\right)$.

It follows that the universal cover of the convex core of a gluing region is the convex hull of $\Lambda\left(\Gamma_{1} \cap \Gamma_{2}\right) \cup P\left(\Gamma_{1}, \Gamma_{2}\right)$. The $\left(\Gamma_{1} \cap \Gamma_{2}\right)$-orbits of points in $P\left(\Gamma_{1}, \Gamma_{2}\right)$ correspond to the thorns forming the spider's legs.

\section{Constructing prefabricated manifolds}

In Proposition 4.1 we construct the pieces that are used to build the prefabricated manifold $Z$. These pieces are submanifolds of covering spaces of the manifold $M$ in Theorem 0.1. The main theorem follows from Theorem 4.2.

Two transverse excellently essential surfaces $J_{1}, J_{2} \subset M$ in a hyperbolic 3-manifold $M=M^{c} \cup \mathcal{V}$ have essential intersections if every component of $J_{1} \cap J_{2} \cap M^{c}$ is either a circle that is not homotopic into $\partial M^{c}$ or an arc that is not homotopic rel endpoints into $\partial M^{c}$.

Proposition 4.1 Suppose $M=M^{c} \cup \mathcal{V}$ is an excellent decomposition of a complete, finite-volume, hyperbolic 3-manifold with cusps and $\partial M^{c}=T_{1} \sqcup \cdots \sqcup T_{p}$. Then there are transverse excellently essential surfaces $\mathcal{J}_{1}, \mathcal{J}_{2} \subset M$ such that every component of these surfaces is QF. Moreover for every torus $T \subset \partial M^{c}$ both $T \cap \mathcal{J}_{1}$ and $T \cap \mathcal{J}_{2}$ are nonempty and have different slopes.

Proof Each homomorphism $\rho: \pi_{1} M \rightarrow \operatorname{SL}(2, \mathbb{C})$ determines a character $\chi: \pi_{1} M \rightarrow \mathbb{C}$ by $\chi(\alpha)=\operatorname{trace}(\rho \alpha)$. The character variety $X$ is the set of all such characters. It is an affine algebraic variety over $\mathbb{C}$. Let $X_{0}$ be the component of $X$ containing the character of the holonomy of the hyperbolic structure on $M$. Thurston [14] proved that $X_{0}$ has complex dimension $p$.

Choose a slope $\alpha_{i}$ on each $T_{i}$. Let $Y=Y\left(\alpha_{1}, \ldots, \alpha_{p}\right)$ be the subset of $X_{0}$ defined by the $p-1$ polynomial equations $\chi^{2}\left(\alpha_{1}\right)=\chi^{2}\left(\alpha_{i}\right)$ for $2 \leq i \leq p$. Then $Y$ is an affine algebraic variety that contains the character $\chi_{0}$ of $\rho_{0}$. This is because at the 
hyperbolic structure every slope is parabolic so $\chi_{0}^{2}(\alpha)=4$ for every slope $\alpha$ on every torus in $\partial M$.

Thus $Y$ has complex dimension at least $p-(p-1)=1$. The function $f=\chi^{2}\left(\alpha_{1}\right)$ is not zero at points on $Y$ close to $\chi_{0}$. This is because a representation $\rho$ close to $\rho_{0}$ with $f=4$ is parabolic on each boundary component. Therefore $\rho$ is the holonomy of a complete finite-volume hyperbolic structure on $M$. By Mostow-Prasad rigidity $\rho$ is conjugate to $\rho_{0}$. Since $Y$ has dimension at least 1 it follows that $Y$ has dimension 1 and $f \neq 4$ in a small deleted neighborhood of $\chi_{0}$ on $Y$ as asserted.

Thus there is a discrete rank- 1 valuation $v$ on $Y$ such that $v(f)<0$. The CullerShalen machinery ([7;8], cf [2, (9.2)]) applied to $(Y, v)$ gives an action of $\pi_{1} M$ on a simplicial tree and an essential surface $\mathcal{J}_{1}=\mathcal{J}\left(\alpha_{1}, \ldots, \alpha_{k}\right)$ dual to this action. Since $v(f)<0$ each $\alpha_{i}$ acts on the tree without a fixed point. Therefore this surface has non-empty intersection with every $T_{i}$ and the slope of $\mathcal{J}_{1}$ on $T_{i}$ is some $\beta_{i} \neq \alpha_{i}$. By surgering annuli, as in [5, Lemma (2.3)], we may arrange that every component of $\mathcal{F}_{1}$ is QF.

Now repeat using $Y=Y\left(\beta_{1}, \ldots, \beta_{p}\right)$. This produces another essential QF surface $\mathcal{J}_{2}=\mathcal{J}\left(\beta_{1}, \ldots, \beta_{p}\right)$ with slope $\gamma_{i} \neq \beta_{i}$ on $T_{i}$. It is routine to show these surfaces can be isotoped to be transverse, and excellently essential.

Theorem 4.2 If $M$ is a complete, finite-volume, hyperbolic 3-manifold with cusps then there is a prefabricated 3-manifold $Z=\mathcal{C} \cup \mathcal{Q}_{1} \cup \mathcal{Q}_{2}$ with a convex thickening $\mathrm{CH}(Z)$ and a local isometry $g: \mathrm{CH}(Z) \rightarrow M$.

Proof Choose an excellent decomposition $M=M^{c} \cup \mathcal{V}$. Let $\mathcal{J}_{1}, \mathcal{J}_{2} \subset M$ be the excellently essential surfaces given by Proposition 4.1. In what follows, $i \in\{1,2\}$. Then $\mathcal{J}_{i}^{c}=\mathcal{J}_{i} \cap M^{c}$ is a compact essential surface in $M^{c}$ and $\partial \mathcal{J}_{i}^{c}$ is a non-empty set of disjoint, essential, simple closed curves. The set of intersection points $\mathcal{M}=\partial \mathcal{J}_{1}^{c} \cap \partial \mathcal{J}_{2}^{c}$ between these curves is finite. Since $\mathcal{J}_{1}$ and $\mathcal{J}_{2}$ each meet every cusp of $M$, and have different slopes, $\mathcal{M}$ contains at least one point on each component of $\partial \mathcal{J}_{i}^{c}$.

Define an equivalence relation on $\{1,2\} \times \mathcal{M}$ by $[i, m]=\left[i^{\prime}, m^{\prime}\right]$ if and only if $i=i^{\prime}$ and both $m$ and $m^{\prime}$ are in the same component of $\mathcal{J}_{i}$. Denote this component $J_{[i, m]}$. Then $Q_{[i, m]}=Q\left(J_{[i, m]}\right)$ is an excellent convex QF manifold and there is a natural projection $f_{[i, m]}: Q_{[i, m]} \rightarrow M$. After thickening if necessary, we may assume there is $q_{i, m} \in Q_{[i, m]}$ with $f_{[i, m]}\left(q_{i, m}\right)=m$. Applying Theorem 3.4 to $\left(M, Q_{[1, m]}, f_{[1, m]}\right)$ and $\left(M, Q_{[2, m]}, f_{[2, m]}\right)$ with base points $q_{1, m}, q_{2, m}$ and $m$ gives an ideal 3-spider $R_{m}$ and two immersed ideal 3-spiders $\left(Q_{[i, m]}, R_{m}, p_{i, m}\right)$.

Define $\mathcal{R}=\left\{R_{m}: m \in \mathcal{M}\right\}$ and $\mathcal{R}_{[i, m]} \subset \mathcal{R}$ to be those ideal 3-spiders that are immersed in $Q_{[i, m]}$. Observe that $\left\{\mathcal{R}_{[i, m]}: m \in \mathcal{M}\right\}$ is a partition of $\mathcal{R}$ for each of $i=1$ and 
$i=2$. There is a wall $W_{[i, m]}=\left(Q_{[i, m]}, \mathcal{R}_{[i, m]}, p_{[i, m]}^{*}\right)$, where $p_{[i, m]}^{*} \mid R_{k}=p_{i, k}$ for each $R_{k} \in \mathcal{R}_{[i, m]}$. Condition (W4) (ample spiders) is satisfied because $\mathcal{M}$ contains at least one point on each component of $\partial \mathcal{J}_{i}^{c}$. Define $\mathcal{W}_{i}=\left\{W_{[i, m]}: m \in \mathcal{M}\right\}$; then $\left|\mathcal{W}_{i}\right|=\left|\mathcal{J}_{i}\right|$. There is a natural homeomorphism $\sigma$ between the ideal spiders in $\mathcal{W}_{1}$ and those in $\mathcal{W}_{2}$ that sends the copy of $R_{m}$ in $W_{[1, m]}$ to the copy in $W_{[2, m]}$. This gives a wall pattern $\left(\mathcal{W}_{1}, \mathcal{W}_{2}, \sigma\right)$.

By Corollary 3.9 each wall $W_{[i, m]}$ is approximated by a connected immersed spider surface $\left(F_{[i, m]}, \mathcal{X}_{[i, m]}, f_{[i, m]}\right)$. Combining these we get two spider surfaces $\mathcal{S}_{1}, \mathcal{S}_{2}$ approximating $\mathcal{W}_{1}$ and $\mathcal{W}_{2}$. Moreover $\sigma$ determines a pairing so we obtain a spider pattern $P=\left(\mathcal{S}_{1}, \mathcal{S}_{2}, \tau\right)$.

Let $d=d(P)>0$ be the constant given by Theorem 2.8 for the spider pattern $P$. Define $\kappa=24 \cdot d \cdot f$, where $f$ is the total number of feet of all the spiders $\bigsqcup_{m} \mathcal{X}_{[1, m]}$. For this value of $\kappa$ there is $\delta_{m}>0$ satisfying Corollary 3.10 for $Q=Q_{[i, m]}$ and all the ideal spiders $R_{m}$ it contains, and $F=F_{[i, m]}$ and all the immersed spiders $\mathcal{X}_{[i, m]}$ it contains. The relative thickenings in Corollary 3.10 of $R_{m}$ are relative to the cusps of $Q_{[i, m]}$ that are the pre-images of $\mathcal{V}$. Now set $\delta=\max \delta_{m}$.

By Theorem 2.8 there is a simple embedded spider pattern $\widetilde{P}^{\delta}=\left(\widetilde{\mathcal{S}}_{1}^{\delta}, \widetilde{\mathcal{S}}_{2}^{\delta}, \tilde{\tau}^{\delta}\right)$ that is a spider cover of spider degree $d$ of a $\delta$-thickening of $P$. Here $\widetilde{\mathcal{S}}_{i}^{\delta}=\left(\tilde{\mathcal{F}}_{i}, \tilde{\mathcal{X}}_{i}^{\delta}, \tilde{f}_{i}^{\delta}\right)$ are the embedded simple spider surfaces of $\widetilde{P}^{\delta}$. The number of spider feet of $\tilde{\mathcal{X}}_{i}^{\delta}$ is $d \cdot f$. Since the cover is simple, $\left|\partial \widetilde{\mathcal{F}}_{1}\right|=d \cdot f=\left|\partial \widetilde{\mathcal{F}}_{2}\right|$.

There are relative $\kappa$-thickenings $\mathcal{W}_{i}^{\kappa}$ of $\mathcal{W}_{i}$ and covers, $\widetilde{\mathcal{W}}_{i}^{\kappa}$, corresponding to $\widetilde{P}^{\delta}$. By the choice of $\delta$ the component walls of $\widetilde{\mathcal{W}}_{i}^{\kappa}$ are simple and the gluing regions are embedded. The pairing determines a bijection between the lifted gluing regions of $\widetilde{\mathcal{W}}_{1}^{\kappa}$ and $\widetilde{\mathcal{W}}_{2}^{\kappa}$. These gluing regions are copies of elements of relative thickenings of components of $\mathcal{R}$. Corresponding gluing regions are isometric. This gives a new wall pattern $\left(\widetilde{\mathcal{W}}_{1}^{\kappa}, \widetilde{\mathcal{W}}_{2}^{\kappa}, \widetilde{\sigma}\right)$. The component walls of this pattern are simple and the gluing regions are disjoint.

We now add back the cusps to the walls. Define $\mathcal{Q}_{i}^{\kappa}$ to be the disjoint union $\bigsqcup_{W} \mathrm{CH}(W)$ over the walls $W$ in $\widetilde{\mathcal{W}}_{i}^{\kappa}$. If we regard the gluing regions as submanifolds of the walls, their convex hulls are ideal 3 -spiders in $\mathcal{Q}_{i}^{\kappa}$, and $\widetilde{\sigma}$ gives a map between these submanifolds of $\mathcal{Q}_{1}^{\kappa}$ and $\mathcal{Q}_{2}^{\kappa}$ that is an isometry on each component. Identifying these submanifolds gives $Y=\mathcal{Q}_{1}^{\kappa} \cup \mathcal{Q}_{2}^{\kappa}$, where $\mathcal{Q}_{1}^{\kappa} \cap \mathcal{Q}_{2}^{\kappa}$ is the union of the ideal 3-spiders.

Then we glue on covers of components of $\mathcal{V}$ to each end of $Y$ to obtain a prefabricated manifold $Z^{\kappa}$. Each rank- 1 cusp of $\mathcal{Q}_{1}$ has been glued to exactly one rank-1 cusp of $\mathcal{Q}_{2}$ along a thorn. These identifications are compatible with the natural projections $f_{[i, m]}$ so there is a local isometry $g: Y \rightarrow M$. 
Each end $E$ of $Y$ is a vertical set: it is the union, $B_{1} \cup B_{2}$, of two vertical rank-1 cusps $B_{i} \subset \mathcal{Q}_{i}$, and $B_{1} \cap B_{2}$ is a thorn. Thus $E$ is diffeomorphic to the product of a ray and a torus minus an open parallelogram. The end $E$ projects into a rank-2 cusp $C \subset \mathcal{V}$. There is a unique finite cover $\widetilde{C}$ of $C$ so that this projection lifts to an isometric embedding. We use this embedding to glue $\widetilde{C}$ onto $E$ and do this for each end $E$ to obtain $Z^{\kappa}$. Define $\mathcal{C}^{\kappa}$ to be the disjoint union of these $\widetilde{C}$. The fact the walls are simple ensures (P1)-(P4), thus $Z^{\kappa}$ is a prefabricated manifold $Z^{\kappa}=\mathcal{Q}_{1}^{\kappa} \cup \mathcal{Q}_{2}^{\kappa} \cup \mathcal{C}^{\kappa}$.

Each component of $\mathcal{Q}_{i}^{\kappa}$ contains at least one gluing region. Each gluing region corresponds to at least 2 spider feet, so $\left|\mathcal{Q}_{i}\right|^{\kappa} \leq d \cdot f$. Also since each component of $\mathcal{C}^{\kappa}$ corresponds to a spider foot so $\left|\mathcal{C}^{\kappa}\right| \leq d \cdot f$. Hence $k:=\left|\mathcal{Q}_{1}^{\kappa}\right|+\left|\mathcal{Q}_{2}^{\kappa}\right|+|\mathcal{C}|-1 \leq 3 \cdot d \cdot f$. Our choice of $\kappa$ above ensures $\kappa \geq 8 k$ as required in Corollary 1.4.

Shrinking the cusps gives a submanifold $\mathcal{C} \subset \mathcal{C}^{\kappa}$ such that $\mathcal{C}^{\kappa}=\operatorname{Th}_{\kappa}(\mathcal{C})$. This gives a prefabricated manifold $Z=\mathcal{Q}_{1} \cup \mathcal{Q}_{2} \cup \mathcal{C}$ contained in $Z^{\kappa}$. Then Corollary 1.4 implies $Z$ has a convex thickening. We remark that $Z$ and $Z^{\kappa}$ might not be connected; however, any component will do.

\section{Comparison with the proof of Masters and Zhang}

The proof in [12] follows the same general outline. This paper is a result of our attempt to understand their proof. They take two (possibly not connected) QF surfaces with boundary and glue together certain finite covers and add covers of cusps. One difference is they produce covers so that the degree of the cover of each component surface is the same. We do not do this, but instead use the condition of simple combinatorics. This approach avoids certain combinatorial problems concerning the compatibility of cyclic orderings of intersection points between two surfaces as one traces around different boundary components of these surfaces. In the approach of Masters and Zhang there is a big distinction depending on whether or not $M$ has only one cusp. In our approach the number of cusps of $M$ plays no role.

We also make use of results from [2], and in particular the convex combination theorem. Masters and Zhang prove and apply a special case of a version of this result. In [11] they introduced a refined version of subgroup separability for a surface with boundary. We found a new proof [3] of a slight generalization of this theorem, and this result is used heavily in this paper. Our proof of the main theorem relies on a study of coverings of surfaces containing certain immersed surfaces, and in particular the spider theorem Theorem 2.5. We wonder if this result about surfaces might find other applications. 


\section{References}

[1] J W Anderson, Intersections of topologically tame subgroups of Kleinian groups, J. Anal. Math. 65 (1995) 77-94 MR1335369

[2] M D Baker, D Cooper, A combination theorem for convex hyperbolic manifolds, with applications to surfaces in 3-manifolds, J. Topol. 1 (2008) 603-642 MR2417445

[3] M D Baker, D Cooper, Conservative subgroup separability for surfaces with boundary, Algebr. Geom. Topol. 13 (2013) 115-125 MR3031638

[4] B H Bowditch, Geometrical finiteness for hyperbolic groups, J. Funct. Anal. 113 (1993) 245-317 MR1218098

[5] D Cooper, D D Long, Some surface subgroups survive surgery, Geom. Topol. 5 (2001) 347-367 MR1825666

[6] D Cooper, D D Long, A W Reid, Essential closed surfaces in bounded 3-manifolds, J. Amer. Math. Soc. 10 (1997) 553-563 MR1431827

[7] M Culler, P B Shalen, Varieties of group representations and splittings of 3-manifolds, Ann. of Math. 117 (1983) 109-146 MR683804

[8] M Culler, P B Shalen, Bounded, separating, incompressible surfaces in knot manifolds, Invent. Math. 75 (1984) 537-545 MR735339

[9] J Kahn, V Markovic, Immersing almost geodesic surfaces in a closed hyperbolic three manifold, Ann. of Math. 175 (2012) 1127-1190 MR2912704

[10] A Kurosch, Die Untergruppen der freien Produkte von beliebigen Gruppen, Math. Ann. 109 (1934) 647-660 MR1512914

[11] J D Masters, X Zhang, Closed quasi-Fuchsian surfaces in hyperbolic knot complements, Geom. Topol. 12 (2008) 2095-2171 MR2431017

[12] J D Masters, X Zhang, Quasi-Fuchsian surfaces in hyperbolic link complements (2009) arXiv:0909.4501v1

[13] T Soma, Function groups in Kleinian groups, Math. Ann. 292 (1992) 181-190 MR1141792

[14] W P Thurston, Three-dimensional manifolds, Kleinian groups and hyperbolic geometry, Bull. Amer. Math. Soc. 6 (1982) 357-381 MR648524

IRMAR, Université de Rennes 1

35042 Rennes, France

Department of Mathematics, University of California, Santa Barbara

Santa Barbara, CA 93106, USA

mark.baker@univ-rennes1.fr, cooper@math.ucsb.edu

Received: 29 June $2014 \quad$ Revised: 21 August 2014 